\title{
An Asymptotic Analysis of Unsteady Diffusion Flames for Large Activation Energies
}

\author{
AMABLE LIÑÁN and ANTONIO CRESPO Instituto Nacional de Técnica Aeroespacial "Esteban \\ Terradas" Madrid
}

\begin{abstract}
The limit of large activation energy is studied for the process of simultaneous mixing and chemical reaction of two reactants undergoing a one-step irreversible Arrhenius reaction. Consideration is restricted to problems of the evolution type-like unsteady mixing and boundary-layer combustion-for which the solution is uniquely determined in terms of the initial conditions. The continuous transition from the nearly-frozen to the near-equilibrium regimes is described. The analysis uncovers the existence of: (i) An ignition regime, in which a mixing layer develops with only minor effects of the chemical reaction, until a thermal runaway occurs somewhere within the mixing region; at this location chemical equilibrium then is established rapidly. (ii) A deflagration regime, in which premixed flames originate from the ignition point and move through the mixing region to burn completely the reactant not in excess. And (iii) a diffusion-flame regime, in which a thin diffusion flame, that is established when the deflagration wave crosses the surface where the reactants are present in stoichiometric proportions, consumes the excess reactants that could not be burned by the premixed flame. This is accomplished by a process in which the reactants diffuse through a thick layer of reaction products. There exists experimental evidence to support this rather complex picture deduced theoretically.
\end{abstract}

\section{NOMENCLATURE}

a Reaction order, Eq. (3)

A parameter defined in Eq. (83)

$b$ reaction order, Eq. (3)

$B$ preexponential factor, Eq. (3)

$c_{p}$ specific heat at constant pressure

$D$ diffusion coefficient

$E$ activation energy

$E_{p}=4 \pi x_{p}^{2} \exp \left(2 \eta_{p}^{2}\right)$

$g$ parameter characterizing the diffusion flame structure, defined in Eq. (85)

$m$ parameter appearing in Eq. (47) characterizing the structure of the reaction zone of premixed flames. It takes different values in different sections

$M$ mean molecular mass

$p$ pressure

$p_{0} \quad$ eigenvalue of Eqs. $(47,48)=(\gamma-\zeta)_{\infty}$

$Q \quad$ heat release per unit mass of fuel

$R$ universal gas constant

$t$ time

$t_{1} \quad$ characteristic chemical time defined in Eq. (13)

$T$ temperature

$T_{a} \quad$ activation temperature $=E / R$

$u_{2}$ velocity component in direction 2 (normal to the initial interface)

w' fuel mass consumption rate $x=(1-\operatorname{erf} \eta) / 2$

$x_{2}$ coordinate in direction 2 (normal to the initial interface)

$y$ reduced mass fraction, introduced in Eq. (14)

$Y$ mass fraction

$z$ non-dimensional material function defined in Eq. (6)

$Z$ stretched coordinate for the diffusion flame, defined in Eq. (82)

\section{Greek Symbols}

a stands for either of $Y_{1} / \nu, Y_{2},-c_{p} T / Q$

$\beta=c_{p}\left(T_{10}-T_{20}\right) / Q Y_{10}$

$\beta_{1}=T_{a}\left(T_{10}-T_{20}\right) / T_{10}{ }^{2}$

$\gamma \quad$ variable proportional to the fuel mass fraction appearing in Eq. (47): it takes different forms in different sections

$\Gamma$ independent variable for the diffusion flame structure, defined in Eq. (81)

$\Delta \quad$ reduced Damköhler number defined by Eq.(86)

$\Delta_{m}$ minimum or extinction value of $\Delta$

$\epsilon \quad$ small parameter $=T_{10} / T_{a}$

$\zeta$ stretched coordinate for the premixed flame structure described by Eq. (47). It takes different forms in different sections of the paper 
$\eta \quad$ similarity variable defined in Eq. (9)

$\eta_{1}$ gives the approximate location of the reaction zone during the ignition stage, given by Eq. (35)

$\Lambda$ Parameter of Eq. (44), defined in Eq. (45). It equals $1 / 2$

$\nu \quad$ stoichiometric mass ratio oxidizer/fuel

$\xi \quad=\beta_{1} x$

$\rho$ density

$\tau$ non-dimensional time $=t / t_{1}$

$\tau_{1}$ non-dimensional time defined in Eq. (37)

$\phi$ non-dimensional temperature increment due to the chemical reaction, defined in Eq. (30)

\section{Subscripts}

0 initial

1 oxidizer

2 fuel

$e$ asymptotic conditions at the infinitely thin diffusion flame

$f$ frozen

$I$ refers to the ingition time and location

$p$ asymptotic conditions at the infinitely thin premixed reaction zone

\section{INTRODUCTION}

The purpose of this paper is to present an asymptotic analysis of the mixing and simultaneous chemical reaction of two reactants undergoing a one step irreversible Arrhenius reaction, when the activation temperature is large compared with the characteristic temperature of the system.

Two types of problems may be encountered in the analysis of chemical reactions in unpremixed systems: (a) Problems of the evolution type, like unsteady mixing and boundary layer combustion without a stagnation point, and (b) Quasi-steady problems like stagnation point boundary layer combustion and quasi-steady droplet combustion. In quasi-steady problems, within a range of Damköhler numbers, multiple solutions exist that correspond to different combustion regimes. In problems of the evolution type the conservation equations are parabolic, because the terms representing the diffusive effects have second order spatial derivatives, while those terms representing the local heating or convective effects are first order with respect to time or with respect to a spatial derivative that does not appear in the diffusive terms. Then, the solution is uniquely determined in terms of the initial and boundary conditions. With increasing values of the time-like variable the flow changes from nearly-frozen, with incipient chemical reaction effects, to near-equilibrium, with a diffusion controlled mode of combustion.

Chung et al. (1966) tried to elucidate by a numerical analysis under which conditions multiple solutions would be obtained in problems of combustion in unpremixed systems, so that a "simple" transition from nearly frozen flow to near-equilibrium flow would not be possible; they failed to recognize the essential difference between quasi-steady and evolution type diffusion flames for which the simple transition is always possible. Lindberg and Schmitz (1969) indicated, in connection with boundary layer problems with surface reactions, how the multiplicity of the steady states is related to the ellipticity of the equations, or some feed-back mechanism in the boundary conditions.

This paper is devoted to the analysis of the continuous transition from frozen flow to nearequilibrium flow in unsteady or evolution type diffusion flames. An asymptotic analysis, for large activation energies, of the regimes appearing in quasi-steady diffusion flames has been presented by Liñán (1973) in a separate paper.

The transition from frozen flow to near-equilibrium flow, for the problem of simultaneous mixing and chemical reaction of two parallel streams of fuel and oxidizer, was analyzed numerically by Liñán (1961), who also carried out a nearly frozen perturbation analysis for the initial mixing region and a near-equilibrium asymptotic analysis of the structure of the thin reaction zone for large distance of the origin of the mixing region, or equivalently for large Damköhler numbers. The mathematically similar problem of the unsteady diffusion and reaction of a substance through another occupying a half space was considered by Pearson (1963) who also obtained the equations describing the structure of the reaction zone for large times.

Liñán (1963) extended to more general diffusion flames his asymptotic analysis of the structure of the reaction zone. Friedlander and Keller (1963) described, independently, the structure of the reaction zone in a steady one dimensional diffusion layer for large Damköhler numbers. Fendell (1965) showed how these asymptotic analysis could be refined by retaining higher order terms with the method of matched asymptotic expansions.

More recent analytical studies specifically devoted to unsteady or boundary layer mixing problems are those of Waldman 't al. (1969) and 
Krishnamurty and Williams (1971) where coordinate expansions are obtained for small or large values of the time-like variable. These expansions may be used to describe the initial nearly frozen mixing, or the asymptotic near-equilibrium conditions.

Mixing and chemical reaction occur also simultaneously, and the resulting equations are again parabolic, in the turbulent mixing of a jet of fuel in a coaxial air stream. A number of numerical studies of this problem have been carried out because of its application to diffusive supersonic combustion; see for example Ferri (1964) and Brevig and Shahrokhi (1971). These studies make use of some turbulent eddy diffusivity and fairly complicated reaction mechanisms. However, their use of laminar kinetics, that is their evaluation of the reaction rates in terms of the local mean values of the concentrations is not justified for these unpremixed systems.

As representative of the diffusion flame problems of the evolution type, we consider here the unsteady mixing of two half spaces of fuel and oxidizer. The problem of the steady mixing of two parallel streams of fuel and oxidizer with equal velocities, leads to the same mathematical problem as that of the unsteady mixing of two half spaces of fuel and oxidizer if the boundary layer assumptions are applicable. The extension of the results to cases where the streams have different velocities is straightforward. The conditions under which the boundary layer assumptions will break down are given in Section VI.

Liebman et al. (1970) have carried out experiments of flame propagation in an unsteady mixing layer that confirm qualitatively the results presented below. Wolanski and Wôjcicki (1973) carried out experiments on the ignition of the unsteady mixing layer that is formed when a combustible gas flows into an oxidizing atmosphere. In order to explain their experimental results, they developed a mathematical model that is similar to the one presented here, and obtained some numerical solutions.

Closely analogous problems are the vaporization and boundary layer combustion of a flat plate of fuel in a stream of oxidizer, as considered by Libby and Economos (1963), Waldman et al. (1969), Krishnamurty and Williams (1971), Kashiwagi and Summerfield (1973) and also the unsteady vaporization, ignition and combustion of a liquid or a solid in a stagnant reacting atmosphere, as treated by Waldman et al. (1969), Strahle (1969), and Hermance and Kumar (1970).
Most of the ideas and results obtained here are applicable to the analysis of ignition and subsequent transition to a diffusion controlled regime in more complex fluid mechanic situations and to more complex reaction mechanisms.

In Section II we shall write the conservation equations for the unsteady mixing case, and indicate how the steady boundary layer mixing of two parallel streams of equal velocities is described by the same equations.

Then we shall show how in the limit of large activation energies for early times (or small Damköhler numbers) mixing occurs with very little chemical reaction effects. However, for appropriately large times one or two regions of frozen flow will coexist within the flow field with regions of equilibrium flow without fuel or without oxidizer. The frozen flow and equilibrium flow regions are separated by thin transition regions which move toward the frozen flow region as premixed flames, burning completely the lean reactant in the mixture formed up to that time, and leave behind a region of local equilibrium, with the excess fuel or oxidizer, mixed with the products of combustion. The two types of regions of equilibrium flow are separated by a thin reaction zone or diffusion flame where the reactants burn in a diffusion-controlled situation.

For large values of the activation energy the premixed flame, that separates the regions of frozen and equilibrium flow, becomes very thin compared to the thickness of the mixing layer, and it may be considered as a discontinuity of the derivatives of the temperatures and the concentrations. If the premixed flame moves sufficiently fast across the mixing layer, the heat conducted from the equilibrium region reaches only a transport layer in the frozen region which is thin compared with the thickness of the mixing layer. The thin reaction zone or premixed flame together with the thin transport zone ahead of it constitute a classical deflagration wave that appears as a surface of discontinuity of the concentration and temperature separating the regions of frozen and equilibrium flow. In these regions heat conduction and mass diffusion can be neglected when the flame is propagating across the mixing layer.

Figure 1 is a schematic showing the evolution of the premixed and diffusion flames separating the frozen and equilibrium flow regions. Figure 1a corresponds to the case where the difference in initial temperatures of fuel and oxidizer is small. The chemical reaction effects in the mixing layer can be neglected in first approximation; however, 

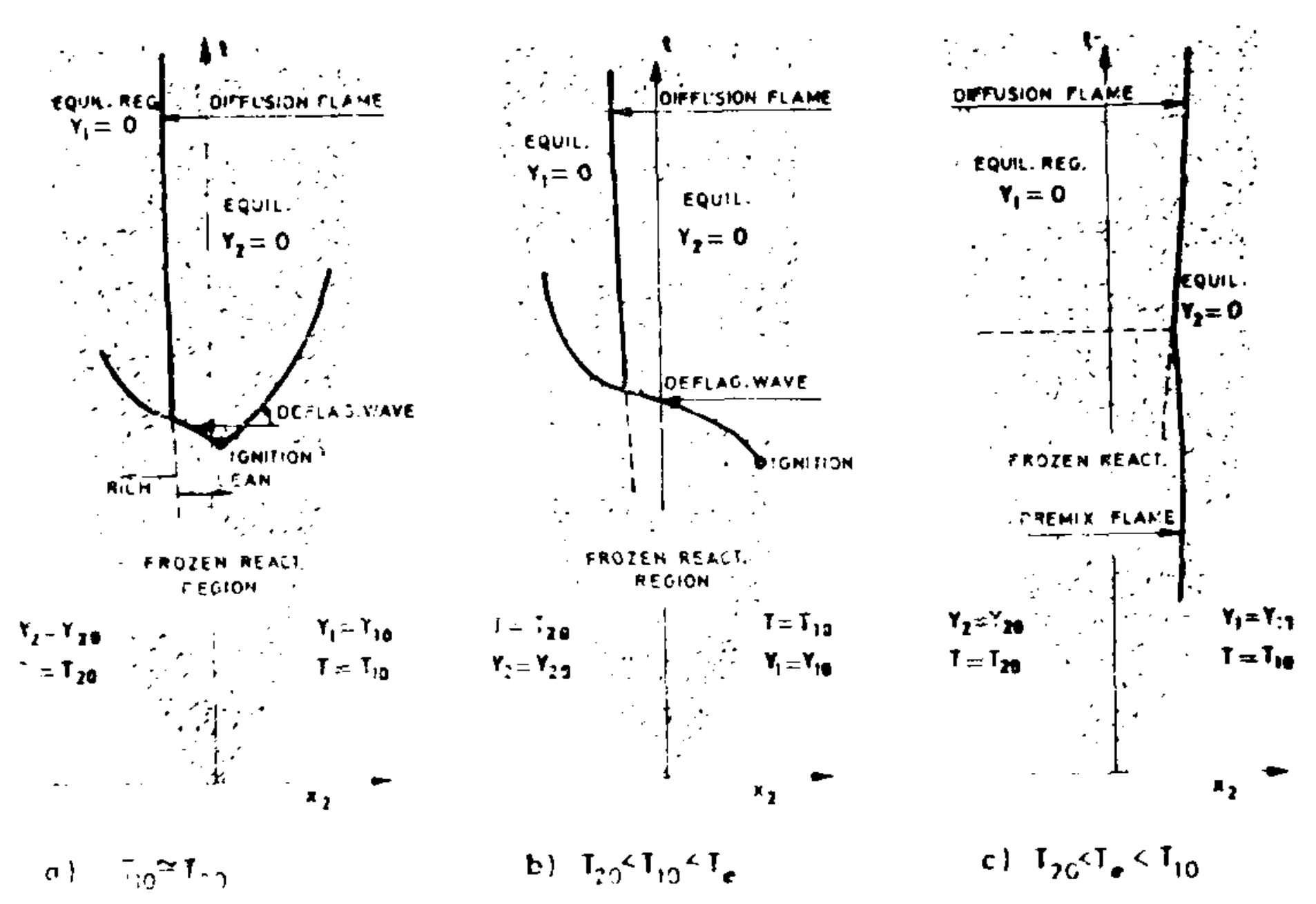

FIGURE 1 Schematic showing the evolution of frozen and equilibrium regions, and the premixed and diffusion flames separating them.

the incipient chemical reaction effects lead to a runaway in temperature at a certain ignition time at a point somewhere in the mixing layer. From this point two deflagration waves move fast through the mixing layer burning to completion the reactant in defect and leaving behind the reaction products and part of the reactant in excess. Two equilibrium regions are established by this process which are separated by a diffusion flame.

Figure $1 \mathrm{~b}$ corresponds to the case where the initial temperatures of fuel and oxidizer are different and lower than the adiabatic diffusion-flame temperature. A deflagration originates at an ignition point in the hot side and propagates across the mixing layer in a short time compared with the ignition time, burning completely the reactant in defect; the two equilibrium regions left behind the deflagration are separated by a diffusion flame.

Figure 1c corresponds to the case for which the initial temperature of the hot reactant is larger than the adiabatic flame temperature. In this case there is not a definite ignition point. The incipient chemical reaction effects appear, as in the previous case, in the hot edge of the mixing layer, leading eventually to a premixed flame that slowly moves across the mixing layer until it becomes a diffusion flame. As a result of the slow motion of the flame the heat released at the flame reaches the whole mixing layer, so that it is not a deflagration wave.

The time evolution of the temperature profiles is also shown in Figure 3 for the three cases considered. The independent coordinate, $x$, is a deformed coordinate normal to the mixing layer to be defined later.

In Section III we carry out a more detailed analysis of the early nearly frozen flow region, so as to show that in a first approximation the flow is chemically frozen; but the incipient chemical reaction effects, appearing in the second approximation, lead after an ignition delay time to thermal runaway, and the subsequent establishment of local equilibrium in a certain point within the mixing region.

In Section IV we analyze the flame propagation process from the regions of equilibrium flow toward the regions of frozen flow where mixing of the reactants had taken place earlier.

In Section $\mathrm{V}$ we shall describe the near-equilibrium diffusion flame structure left behind by the premixed flame when it crosses the surface where the reactants were in stoichiometric proportions.

In Section VI we indicate how upstream diffusion has to be taken into account in the analysis of the parallel mixing case when the premixed flame velocity is of the order of, although smaller than the flow velocity. Upstream diffusion was retained in an analysis by Clarke $(1967 \mathrm{a}, \mathrm{b})$ of near-equilibrium diffusion flames by using the Oseen approximation for the conservation equations.

The discussion and generalization of the results is given in Section VII.

\section{FORMULATION}

The equations that describe the unsteady onedimensional mixing and simultaneous chemical reaction of two half spaces of fuel and oxidizer are:

\section{Continuity}

$$
\frac{\partial \rho}{\partial t}+\frac{\partial \rho u_{2}}{\partial x_{2}}=0
$$

where $\rho$ is the density, $t$ the time. For convenience we define a coordinate system in which the origin is the fluid or material surface that coincides at $t=0$ with the original fuel-oxidizer interface and $u_{2}$ and $x_{2}$ are the velocity and coordinate normal to this fluid surface. As a result of this choice $u_{2}=0$ at $x_{2}=0$. For any other choice of the coordinate system this fluid surface will have a velocity and position whose determination will require an analysis of the flow in the regions exterior to the mixing layer; however, we do not need to carry out this analysis because, under the assumptions made below, the structure of the mixing region does not depend on the transverse motion of the fluid surface mentioned above. 
Species and Energy Conservation

$$
\frac{\partial \alpha}{\partial t}+u_{2} \frac{\partial a}{\partial x_{2}}=\frac{1}{\rho} \frac{\partial}{\partial x_{2}}\left(\rho D \frac{\partial a}{\partial x_{2}}\right)-\frac{w}{\rho}
$$

where $\alpha$ stands for $Y_{1} / \nu, Y_{2}$ or $-c_{p} T / Q$ and $w$ is the overall mass rate of consumption of fuel, given by

$w / \rho=M_{2} B\left(\rho Y_{1} / M_{1}\right)^{a}\left(\rho Y_{2} / M_{2}\right)^{b} \exp \left(-T_{a} / T\right)$

for a one-step irreversible Arrhenius reaction, of preexponential factor $B$, activation temperature $T_{a}$, and reaction orders $a$ and $b ; Q$ is the heat release per unit mass of fuel, and $v$ is the stoichiometric mass ratio oxidizer/fuel. The activation temperature $T_{a}$ equals $E / R$ where $E$ is the activation energy and $R$ the universal gas constant. Although the preexponential factor, $B$, is considered constant in this paper, the results are also applicable to cases where $B$ is a slowly varying function of $T, Y_{1}$ and $Y_{2}$, without zeroes in the range of variation of these variables. $M_{1}$ and $M_{2}$ are the molecular masses.

In order to obtain Eq. (2) as the conservation equation for the reacting species and the temperature, we must assume equal diffusivities of heat and mass $D$, and equal and constant specific heats $c_{p}$. We in addition neglect the kinetic energy associated with the transverse flow when compared with the changes in thermal energy. We neglect also the spatial and time variations in pressure required to produce the transverse velocity field, which implies that the mixing region is unconfined. Because we consider $p$ to be constant we shall not make use of the momentum equation. $\dagger$

\section{Equation of State}

$$
p=(R / M) \rho T
$$

where $p$ is the pressure, $R$ is the universal gas constant and $M$ is the local mean molecular weight, which is a function of the mass fractions. We shall, consistently with the previous assumptions, use a constant mean value for the mean molecular weight $M$.

We should add to Eqs. (1) to (4) a relation giving the diffusion coefficient $D$ in terms of the temperature, pressure and concentrations. However, we shall assume below that $\rho^{2} D$ is a constant, as this will not affect essentially the results but will simplify the mathematical analysis.

† As a result of this assumption we neglect the effects of pressure waves that could eventually generate a detonation wave.
As initial and boundary conditions we shall take

$$
\begin{aligned}
& Y_{1}=\begin{array}{r}
Y_{10}, \quad Y_{2}=0, \quad T=T_{10} \text { at } t=0, \\
x_{2}>0 \text { and at } t>0, \quad x_{2} \rightarrow \infty,
\end{array} \\
& Y_{1}=0, \quad Y_{2}=Y_{20}, \quad T=T_{20} \text { at } t=0 \text {, } \\
& x_{2}<0 \text { and at } t>0, \quad x_{2} \rightarrow-\infty
\end{aligned}
$$

The above system of equations and subsidiary conditions will determine the temperature, density and concentration fields for times, $t$, larger than 0 .

We should indicate at this stage that the same mathematical problem is obtained when analyzing the process of mixing and simultaneous chemical reaction between two streams one with oxidizer of mass fraction $Y_{10}$ and temperature $T_{10}$ and the other with a mass fraction of fuel $Y_{20}$ and a temperature $T_{20}$. They both flow originally in the $x_{1}$ direction with equal velocity $V$ and are allowed to mix for $x_{1}>0$.

If the Peclet number $V L / D$, based on the charateristic combustor length $L$, is large, the boundary layer approximations may be used for the analysis of the mixing process. This means that we may neglect diffusion in the upstream direction and in addition consider the pressure constant.

The momentum equation in the $x_{1}$ direction, if we neglect the effect of the boundary layers in the separation plate, shows that the velocity component $v_{1}$ is constant $v_{1}=V$.

The continuity equation and the species and energy conservation equations take then the form of Eqs. (1) and (2) if we neglect the kinetic energy associated with the transverse flow compared with the thermal energy. The variable $t$ stands now for $x_{1} / V$.

We thus reproduce Eqs. (1) to (4) as describing this parallel mixing problem. The conditions for the validity of the approximations involved as well as the extension to cases with different velocities are left for Section VI. See also Williams (1965).

Because of the constancy of $\rho^{2} D$ we may use a Howarth-Dorodnitzyn transformation to reduce Eq. (2) to an incompressible form. Let us introduce the variables

$$
\tau=t / t_{1}
$$

and

$$
z=\left(\rho^{2} D t_{1}\right)^{-1 / 2} \int_{0}^{x_{2}} \rho d x_{2}
$$

where $t_{1}$ is a chatacreristic time which we shall choose later, and $z$ is a nondimensional material 
function originating from the continuity equation (1). In terms of these variables Eq. (2) takes the form of the heat conduction equation

$$
\frac{\partial \alpha}{\partial \tau}-\frac{\partial^{2} \alpha}{\partial z^{2}}=-t_{1} \frac{w}{\rho}
$$

Equation (7) may also be written as

$$
\frac{\partial \alpha}{\partial \tau}-\frac{\eta}{2 \tau} \frac{\partial \alpha}{\partial \eta}-\frac{1}{4 \tau} \frac{\partial^{2} \alpha}{\partial \eta^{2}}=-t_{1} w / \rho
$$

in terms of the variables $\tau$ and $\eta$, where

$$
\eta=z / 2 \sqrt{ }(\tau)
$$

is the similarity variable for the chemically frozen heat conduction. That is, if we integrate Eqs. (7) or (8) without the source term, subject to the subsidiary conditions (5), we obtain the frozen flow temperature and concentration distributions

$$
\begin{aligned}
& Y_{1}=Y_{1 f}=Y_{10}(1+\operatorname{erf} \eta) / 2 \\
& Y_{2}=Y_{2 f}=Y_{20}(1-\operatorname{erf} \eta) / 2 \\
& T=T_{f}=T_{10}+\left(T_{20}-T_{10}\right)(1-\operatorname{erf} \eta) / 2
\end{aligned}
$$

When treating the reacting case, because of the assumption made of equal diffusivities of mass and heat, by using the Schvab-Zeldovich procedure, we may further reduce the unsteady mixing and chemical reaction problem to the solution of just one partial differential equation, the heat conduction equation for $T$ with a source term, which is a nonlinear function of $T$ and $\eta$. To do this, we first notice that the combinations $Y_{1} / \nu+c_{p} T / Q$ and $Y_{2}+c_{p} T / Q$ do satisfy the heat conduction equation (8) without source terms. So that when the conditions (5) are taken into account, we obtain

$$
\begin{gathered}
Y_{1} / \nu+c_{p} T / Q=Y_{1 f} / \nu+c_{p} T_{f} / Q \\
Y_{2}+c_{p} T / Q=Y_{2 f}+c_{p} T_{f} / Q
\end{gathered}
$$

where the terms in the right hand side are functions of $\eta$ given by Eq. (10).

The relations (11) and (12) giving the concentration distributions in terms of $T$ and $\eta$, enable us to reduce our problem to obtaining the solution of the heat conduction equation with a source term which is a nonlinear known function of $T$ and $\eta$. This equation is obtained from Eq. (7), taking Eqs. (3), (11) and (12) into account. If we choose for $t_{1}$ a characteristic chemical time based on the maximum initial temperature, which for definiteness we assume to be $T_{10}$,

$$
\begin{aligned}
t_{1}= & \frac{1}{M_{2} B}\left(\frac{M_{1}}{\rho_{10} Y_{10}}\right)^{a}\left(\frac{M_{2}}{\rho_{10} Y_{20}}\right)^{b} \\
& \times \frac{c_{p} T_{10}}{Q} \frac{T_{10}}{T_{a}} \exp \left(T_{a} / T_{10}\right),
\end{aligned}
$$

we obtain,

$$
\begin{aligned}
\frac{\partial T}{\partial \tau} & -\frac{\partial^{2} T}{\partial z^{2}} \\
& =\frac{T_{10}^{2}}{T_{a}}\left(\frac{T_{10}}{T}\right)^{a+b} y_{1}{ }^{a} y_{2}^{b} \exp \left[\frac{T_{a}}{T_{10}} \frac{T-T_{10}}{T}\right]
\end{aligned}
$$

In Eq. (14) the reduced concentrations $y_{1}=Y_{1} / Y_{10}$ and $y_{2}=y_{2} / Y_{20}$ are given, according to Eqs. (11) and (12), by

$$
\begin{aligned}
& y_{1}=1-x-x \nu c_{p}\left(T_{10}-T_{20}\right) / Q Y_{10}- \\
& v c_{p}\left(T-T_{10}\right) Q Y_{10} \\
& y_{2}=x-x c_{p}\left(T_{10}-T_{20}\right) / Q Y_{20}-c_{p}\left(T-T_{10}\right) / Q Y_{20}(16)
\end{aligned}
$$

where

$$
x=(1-\operatorname{erf} \eta) / 2 .
$$

Equation (14) should be solved with the subsidiary conditions

$T=T_{10}$ for $\tau=0, z>0$, and for $\tau>0, z \rightarrow \infty$

and

$T=T_{20}$ for $\tau=0, z<0$, and for $\tau>0, z \rightarrow-\infty$

An important conclusion that can be obtained from this formulation is that the temperature and concentration profiles for different values of the diffusion and heat conduction coefficients are similar. The thickness of the mixing layer is proportional to the square root of the diffusion coefficients as can be seen from Eq. (6). The time is scaled with a characteristic chemical time (Eq. (13)). Thus, the time of thermal runaway or ignition time will be independent of the transport coefficients, although a dependence with the Lewis number would appear if we did not consider equal diffusivities of heat and mass.

Because the source term in Eq. (14) is a function of $T$ and $\eta$ (or $x$ ) there is some advantage in writing the heat conduction equation (14) in terms of the 
variables $\tau$ and $\eta$ as

$$
\begin{aligned}
& \frac{\partial T}{\partial \tau}-\frac{\eta}{2 \tau} \frac{\partial T}{\partial \eta}-\frac{1}{4 \tau} \frac{\partial^{2} T}{\partial \eta^{2}} \\
& =\frac{T_{10}^{2}}{T_{a}}\left(\frac{T_{10}}{T}\right)^{a+b} y_{1}^{a} y_{2}^{b} \exp \left(\frac{T_{a}}{T_{10}} \frac{T-T_{10}}{T}\right)
\end{aligned}
$$

or in terms of the variables $\tau$ and $x$ if we substitute the left hand side of Eq. (18) by

$$
\frac{\partial T}{\partial \tau}-\frac{\exp \left(-2 \eta^{2}\right)}{4 \pi \tau} \frac{\partial^{2} T}{\partial x^{2}}
$$

The boundary conditions are

and

$$
\begin{array}{lll}
T=T_{10} & \text { for } \quad \tau>0, \quad \eta \rightarrow \infty \\
T=T_{20} & \text { for } \quad \tau>0, \quad \eta \rightarrow-\infty
\end{array}
$$

which takes into account both the initial and boundary conditions (17), if we add the condition that $T$ and its first and second derivatives with respect to $\eta$ be continuous at $\tau=0$. When the variable $\eta$ is replaced by $x$, the limit $\eta \rightarrow \pm \infty$ correspond to $x=0$ and $x=1$, respectively.

For small times

$$
T=T_{f}(\eta)+\tau F_{1}(\eta)+\tau^{2} F_{2}(\eta)+\ldots
$$

where $T_{f}(\eta)$ is the frozen flow temperature distribution

$$
T_{f}=T_{10}+\left(T_{20}-T_{10}\right)(1-\operatorname{erf} \eta) / 2
$$

and $F_{1}(\eta)$ is the solution of the differential equation, obtained by introducing Eq. (20) into Eq. (18),

$$
\begin{aligned}
& F_{1}-\eta F_{1}^{\prime} / 2-F_{1}^{\prime \prime} / 4=\frac{T_{10}^{2}}{T_{a}}\left(\frac{T_{f}}{T_{10}}\right)^{-(a+b)} \\
& \underset{y_{1 f}}{a} y_{2 f}^{b} \exp \left(\frac{T_{a}}{T_{10}} \frac{T_{f}-T_{10}}{T_{f}}\right)
\end{aligned}
$$

to be solved with the boundary conditions

$$
F_{1}(-\infty)=F_{1}(+\infty)=0
$$

The temperature at fixed $\eta$ is seen to increase, for small values of time $\tau$, linearly with $\tau$, according to Eq. (20).

The expansion (20) is not convergent for large values of time, when according to Eq. (18), chemical equilibrium should be approached. Chemical equilibrium means that $y_{1}=0$ on one side of a thin reaction region and $y_{2}=0$ on the other. That is:

For $\eta<\eta_{e}$, or $x>x_{e}$ :

or

$$
y_{1}=0, \quad T=T_{f}+Q Y_{1 f} / \nu c_{p}
$$

$$
T=T_{20}+(1-x)\left(Q Y_{10} / \nu c_{p}+T_{10}-T_{20}\right)
$$

For $\eta>\eta_{e}$ or $x<x_{e}$

$$
y_{2}=0, \quad T=T_{f}+Q Y_{2 f} / c_{p}
$$

or

$$
T=T_{10}+x\left(Q Y_{20} / c_{p}-T_{10}+T_{20}\right)
$$

where $\eta_{e}$ (or $x_{e}$ ) gives the location of the thin flame where the concentration of both reactants vanishes and $T$ takes the adiabatic flame value, $T_{e}$;

$$
T_{e}=T_{10}+\left(Q Y_{20} / c_{p}-T_{10}+T_{20}\right) Y_{10} /\left(Y_{10}+\nu Y_{20}\right)
$$

and

$$
x_{e}=\left(1-\operatorname{erf} \eta_{e}\right) / 2=Y_{10} /\left(Y_{10}+\nu Y_{20}\right)
$$

If we would plot, see Figure 2, the temperature, at $\eta=\eta_{e}$ for example, as a function of time we would get a continuous transition from the frozen flow value $T_{f}\left(\eta_{e}\right)$ to the adibatic flame value $T_{e}$. It is the purpose of the present work to analyze this transition by obtaining the solution of Eq. (18) with the boundary condition (19), or equivalently the solution of Eq. (14) with the subsidiary conditions (17), for large values of the non-dimensional activation energy $T_{a} / T_{10}$, and fixed values, in principle of order unity, of all the other parameters in the system.

The fact that the ratio $T_{a} / T_{10}$ appears as a factor in the Arrhenius exponent in Eq. (18) makes it clear

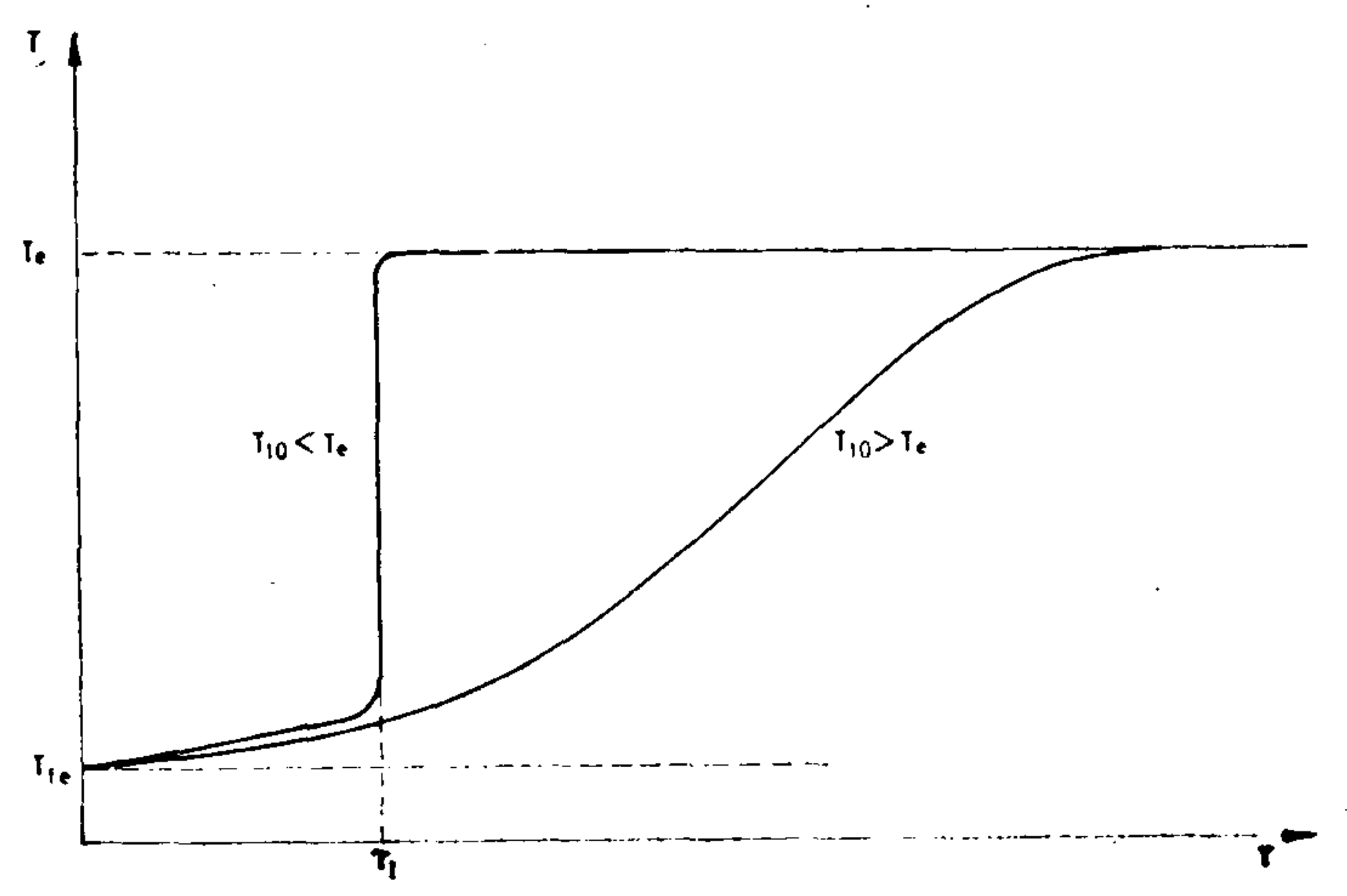

FIGURE 2 Schematic showing the transition of the temperature from its frozen to its adiabatic flame value for different values of $T_{10}$. 
that the chemical production term will become exponentially large for $T>T_{10}$ and exponentially small for $T<T_{10}$ unless the difference $T-T_{10}$ is so small as to make $T-T_{10}$ of order $T_{10}^{2} / T_{a}$ or $y_{1}=0$ or $y_{2}=0$.

It is then clear from Eqs. (20) and (22) that for small times a small linear increment in temperature above the frozen flow value, $T-T_{10} \sim \tau T_{10}^{2} / T_{a}$, is obtained with a correspondingly small effect on the reactant concentration. However, higher order terms in the expansion (20) have to be retained when $\tau$ reaches order unity because of the large effect of the Arrhenius exponent in the reaction rates when $T_{a} /\left(T-T_{10}\right) T_{10}{ }^{2}$ becomes of order unity, or larger. The reaction rates then become so fast, and thereby the rate of heat release so large, that suddenly a thermal runaway leading to the establishment of local chemical equilibrium, occurs somewhere in the mixing region. The choice of $t_{1}$, as given by Eq. (13), as the characteristic time, was made so as to have the ignition event occurring at an ignition time, $\tau_{I}$, of order unity. The factor $T_{10} / T_{a}$ was left in Eq. (18) so that at $\tau \sim 1$ the increment in temperature above the frozen flow value was of order $T_{10}^{2} / T_{a}$, as required to initiate the thermal runaway.

As indicated before, the limiting forms of Eq. (18) for $T_{10} / T_{a} \rightarrow 0$ and $\tau$ of order unity are, if we anticipate that the derivatives of $T$ are of order unity,

Frozen flow

$$
T=T_{f}
$$

or Equilibrium flow with

$$
y_{1}=0
$$

or Equilibrium flow with

$$
y_{2}=0
$$

We shall show below, that, within the mixing layer, regions of equilibrium flow may coexist with regions of frozen flow.

When chemical equilibrium is established locally by the thermal runaway process, either $y_{1}$ or $y_{2}$ become zero at that location and the temperature increases suddenly from a nearly frozen value to that given by Eqs. (23) or (24).

Chemical equilibrium then extends to the other nearly frozen regions of the mixing layer by means of a deflagration wave mechanism, see Figures 1 and 3. Heat conducted from the region of chemical

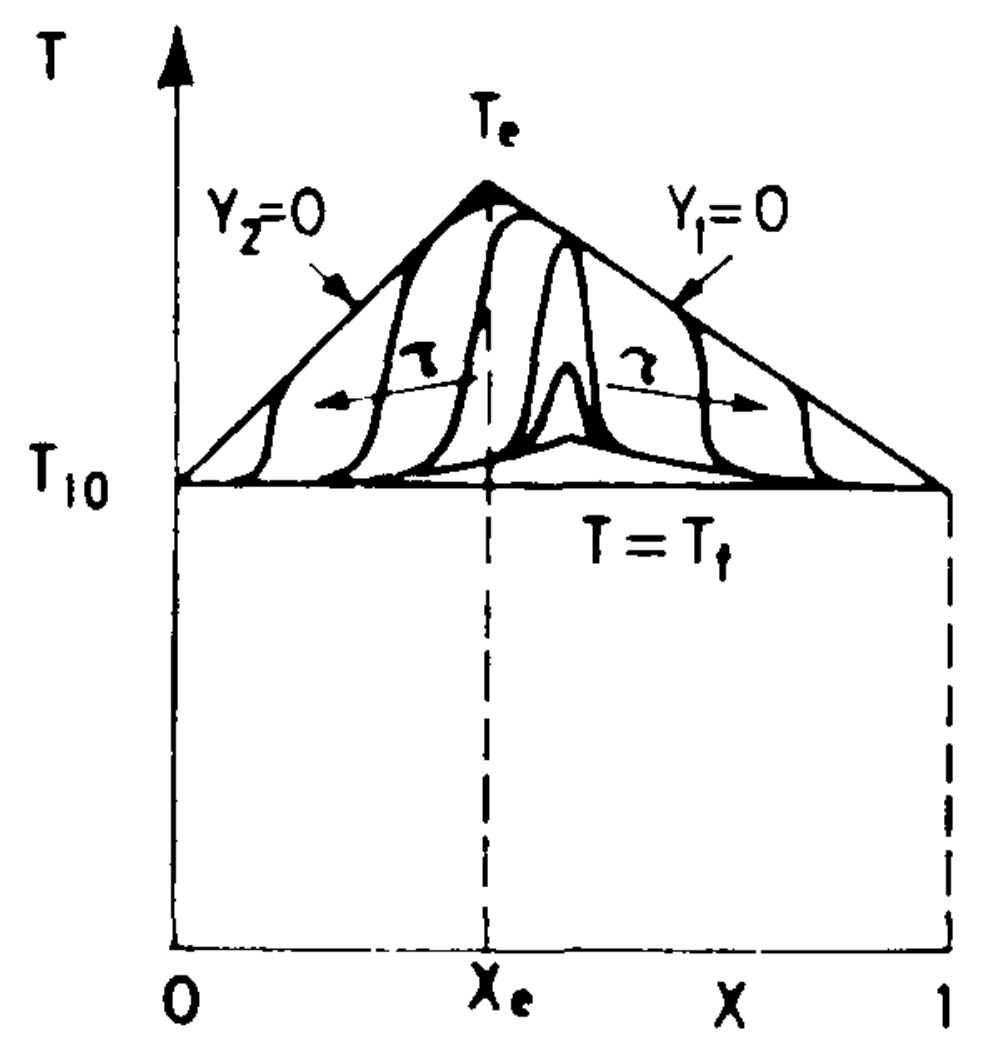

$$
B_{1} \sim 1(a)
$$

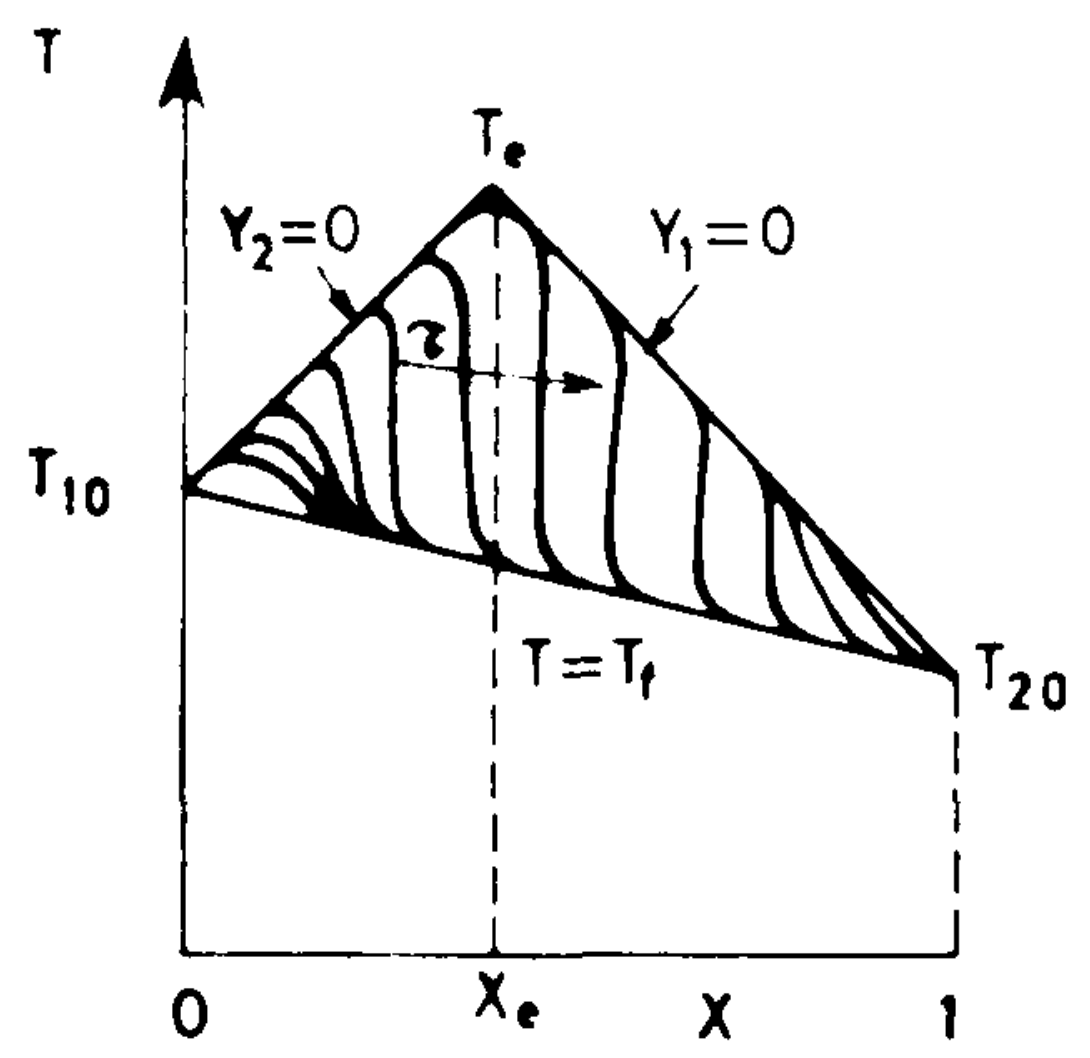

$B<1$ (b)

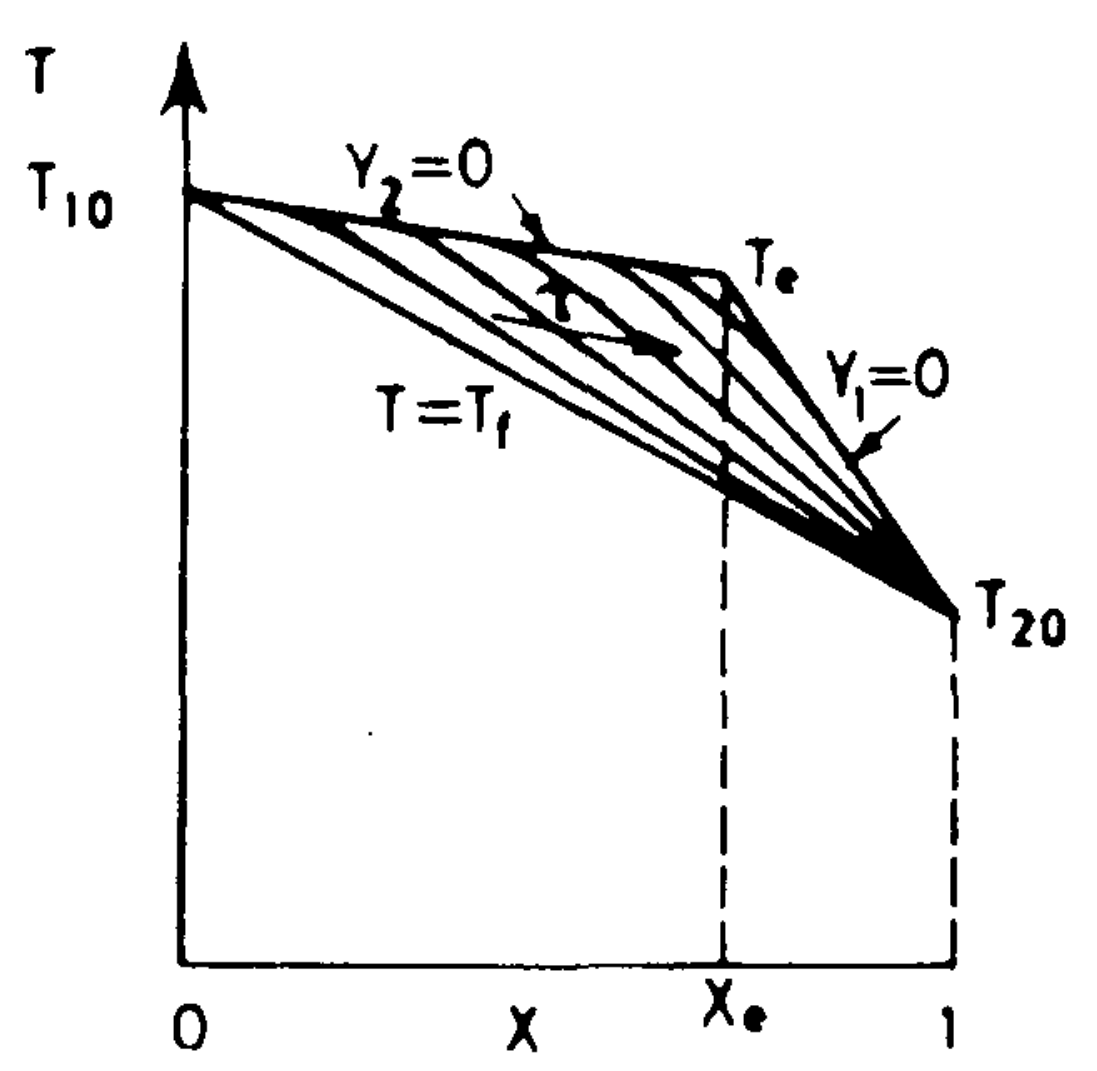

$B>1(c)$

FIGURE 3 Schematic showing the evolution of the temperature profiles.

equilibrium to the frozen flow regions of lower temperature increases the temperature, and consequently the reaction rates, so that equilibirium is finally reached in a region which advances toward the frozen flow region at a well defired speed.

We shall see below how two deflagration waves of small thickness compared with the thickness of the mixing layer, originate at the ignition plane and move across the mixing layer in a time small compared with the ignition time. These deflagration waves, or premixed flames, locally consume one of the reactants leaving behind the products of the reaction, together with the fraction of the reactant that was in excess. When the deflagration wave crosses the surface where the reactants were in 
stoichiometric proportions $\left(\eta=\eta_{e}\right.$ in our case), a thin diffusion flame is established, where the reactants that were not burned by the premixed flame are consumed after diffusing across the layer of products of the reaction.

We shall first analyze in Section III the ignition period where the incipient effects of chemical reaction lead eventually to a thermal runaway. We shall then analyze in Section IV the structure of the premixed flame or transition region between the regions of frozen flow and equilibrium flow. From the analysis of the structure of the premixed flame we shall obtain the local premixed flame propagation velocity which will enable us to calculate the trajectory of these flames in the $\left(x_{2}, t\right)$ plane.

\section{IGNITION STAGE}

Small relative temperature increments, $\left(T-T_{10}\right) /$ $T_{10}$, of order $T_{10} / T_{a}$ suffice to increase the reaction rate by a large factor and thereby produce the precipitous rise in temperature leading to local equilibrium. To show this we shall write the exponential Arrhenius factor in the form

$\exp \left(-T_{a} / T\right)=\exp \left(-T_{a} / T_{10}\right) \exp \left\{T_{a}\left(T-T_{10}\right) / T_{10} T\right\}$

For small temperature increments, $\left(T-T_{10}\right) / T_{10}$, of order $T_{10} / T_{a}$, the argument in curly brackets of the last exponential is of order unity, and the reaction rate changes from its value at the temperature $T_{10}$ by an important factor. (For example, this factor is 20 for $\left.\left(T-T_{10}\right) / T_{10}=3 T_{10} / T_{a}.\right)$

To describe the ignition stage we shall therefore write Eq. (14) in terms of the variable

$$
\phi=\left(T-T_{f}\right) T_{a} / T_{10}
$$

We thus obtain

$$
\begin{aligned}
\frac{\partial \phi}{\partial \tau}- & \frac{\partial^{2} \phi}{\partial z^{2}}=\left(\frac{T_{f}}{T_{10}}+\epsilon \phi\right)^{-(a+b)} \\
& \times y_{1}^{a} y_{2}^{b} \exp \frac{\phi+\epsilon^{-1}\left(T_{f} / T_{10}-1\right)}{T_{f} / T_{10}+\epsilon \phi}
\end{aligned}
$$

where $\epsilon$ is the small parameter $T_{10} / T_{a}$, and

$$
\begin{aligned}
y_{1} & =1-x-\epsilon \phi c_{p} T_{10} v / Q Y_{10} \\
y_{2} & =x-\epsilon \phi c_{p} T_{10} / Q Y_{20} \\
T_{f} / T_{10} & =1-\left(T_{10}-T_{20}\right) x / T_{10}
\end{aligned}
$$

where $x=(1-\operatorname{erf} \eta) / 2$.
This equation is to be solved with the initial conditions $\phi=0$ at $\tau=0$, and the boundary conditions $\phi=0$ at $z \rightarrow \pm \infty$.

The negative term $\epsilon^{-1}\left(T_{f} / T_{10}-1\right)$ in the Arrhenius exponent is indicative of the fact that the chemical reaction will be quenched for $z$ such that $T_{f} / T_{10}$ is well below 1. Unless the initial temperature difference of both reactants is small enough to make

$$
\beta_{1}=\left(1-T_{20} / T_{10}\right) / \epsilon
$$

of order unity, the chemical reaction will be confined in the ignition stage to a thin region where the frozen temperature is high, close to $T_{10}$. We shall begin with the analysis of the case when $\beta_{1} \sim 1$.

\section{Ignition for Nearly Equal Initial Temperatures}

If in Eq. (31) we now take the limit $\epsilon \rightarrow 0$, for fixed $\beta_{1}$ of order unity, we obtain the equation

$$
\frac{\partial \phi}{\partial \tau}-\frac{\partial^{2} \phi}{\partial z^{2}}=x^{b}(1-x)^{a} \exp \left[\phi-\beta_{1} x\right]
$$

if we anticipate that $\phi$ and its derivatives remain finite in this limit process.

We could also obtain Eq. (33) by neglecting terms of order $\epsilon$ in Eq. (31); or by inserting in Eq. (31) an expansion of $\phi$ in terms of $\epsilon$ and equating terms of equal order in $\epsilon$. Notice that Eq. (33) results from calculating in the energy equation the reaction rate in terms of the frozen reactant concentrations and by linearizing the Arrhenius exponent around the temperature $T_{10}$.

Equation (33) gives the temperature increment above the frozen flow value in terms of the variables

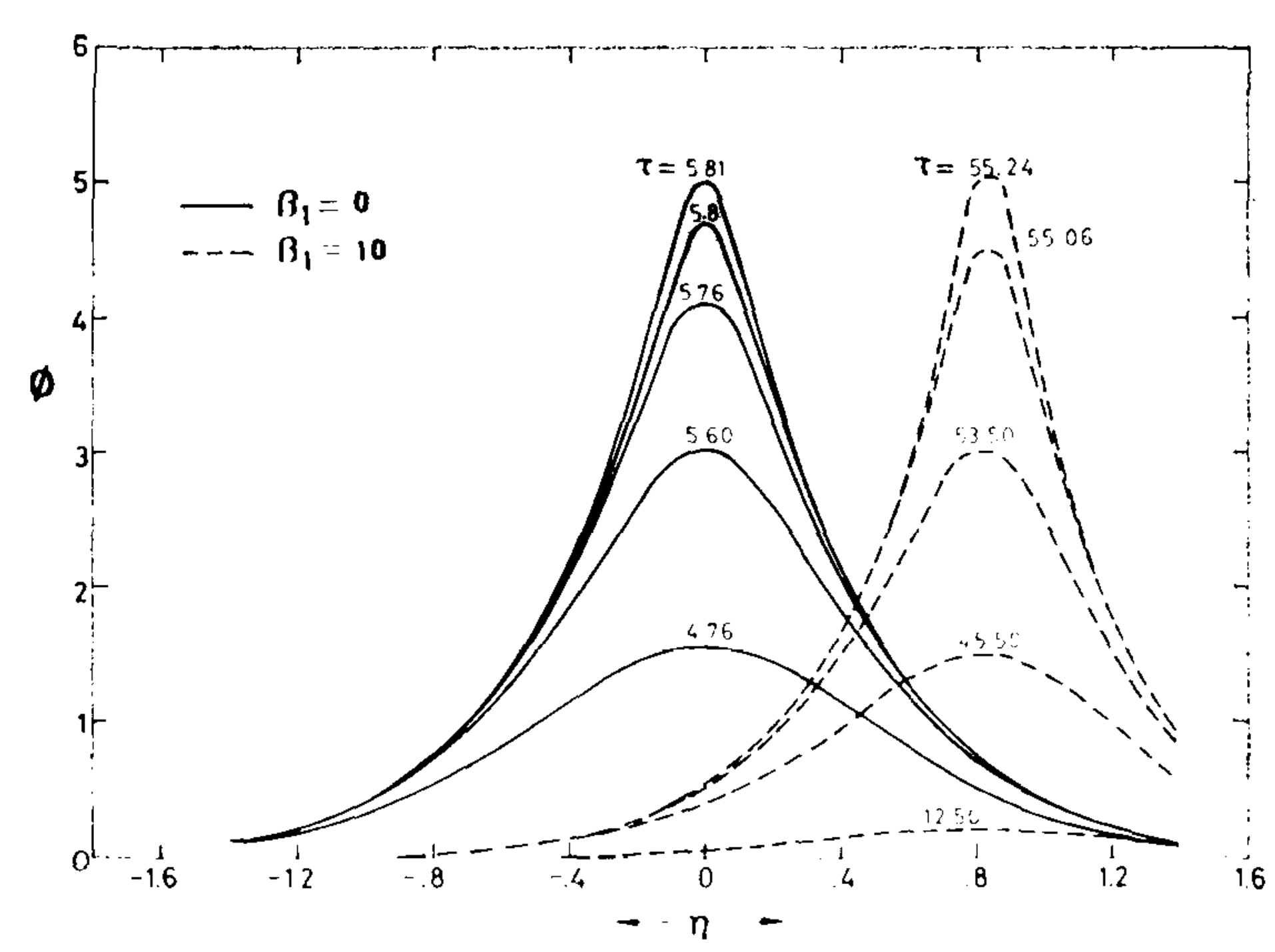

FIGURE 4 Non-dimensional temperature increments due to chemical reaction during the ignition stage for small initial differences in temperature. 


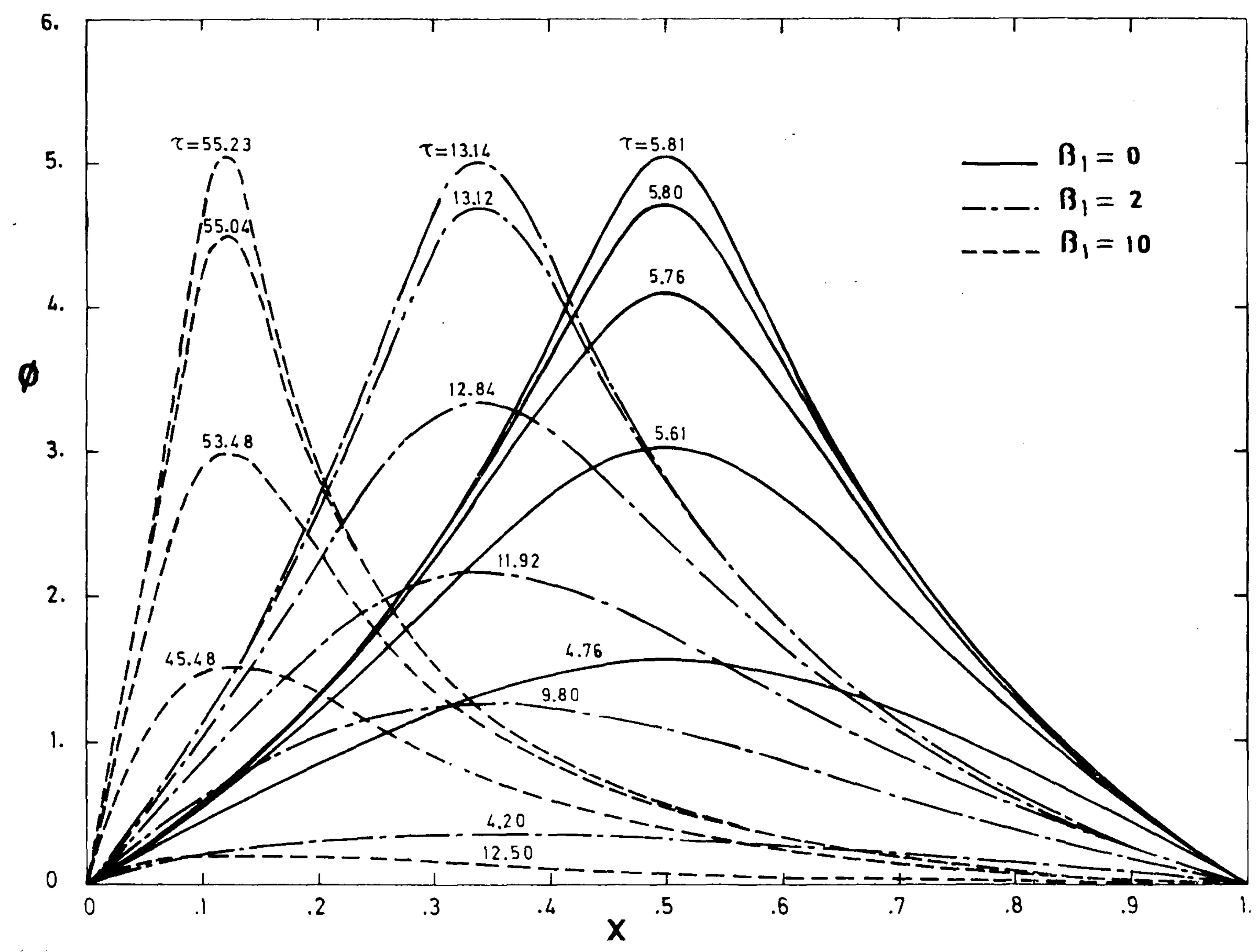

FIGURE 5 Same as in Figure 4, in terms of the variable $x=(1-\operatorname{erf} \eta) / 2$.

$\tau$ and $z$. Eq. (33), or more exactly the equation with the heat conduction operator written in terms of the variables $\tau$ and $\eta$, was numerically integrated for several values of $\beta_{1}$ and $a=b=1$. Some of the resulting profiles $\phi(\eta, \tau)$ or $\phi(x, \tau)$ are shown in Figure 4 and 5.

The temperature increment $\phi$ becomes infinite at $\tau=\tau_{I}$ and $\eta=\eta_{I}$ thereby determining the ignition delay and ignition spot. Figure 6 shows how $\phi$ increases with $\tau$ at $\eta=\gamma_{I I}$, and in Figure 7, $\tau_{I}$ and $x_{I}$ are shown as a function of $\beta_{1}$. When $\phi$ becomes large compared with 1 , the neglected terms in Eq. (31) will become important and Eq. (33) and its solution will not adequately represent the temperature distribution. However this occurs at $\tau$ so close to $\tau_{I}$ that the ignition delay will be only slightly modified by this term. The infinite in $\phi$ is due to the linearization of the Arrhenius exponent; instead, the temperature $\phi$, as given by the solution of Eq. (31), does not increase to infinity when $\tau$ and

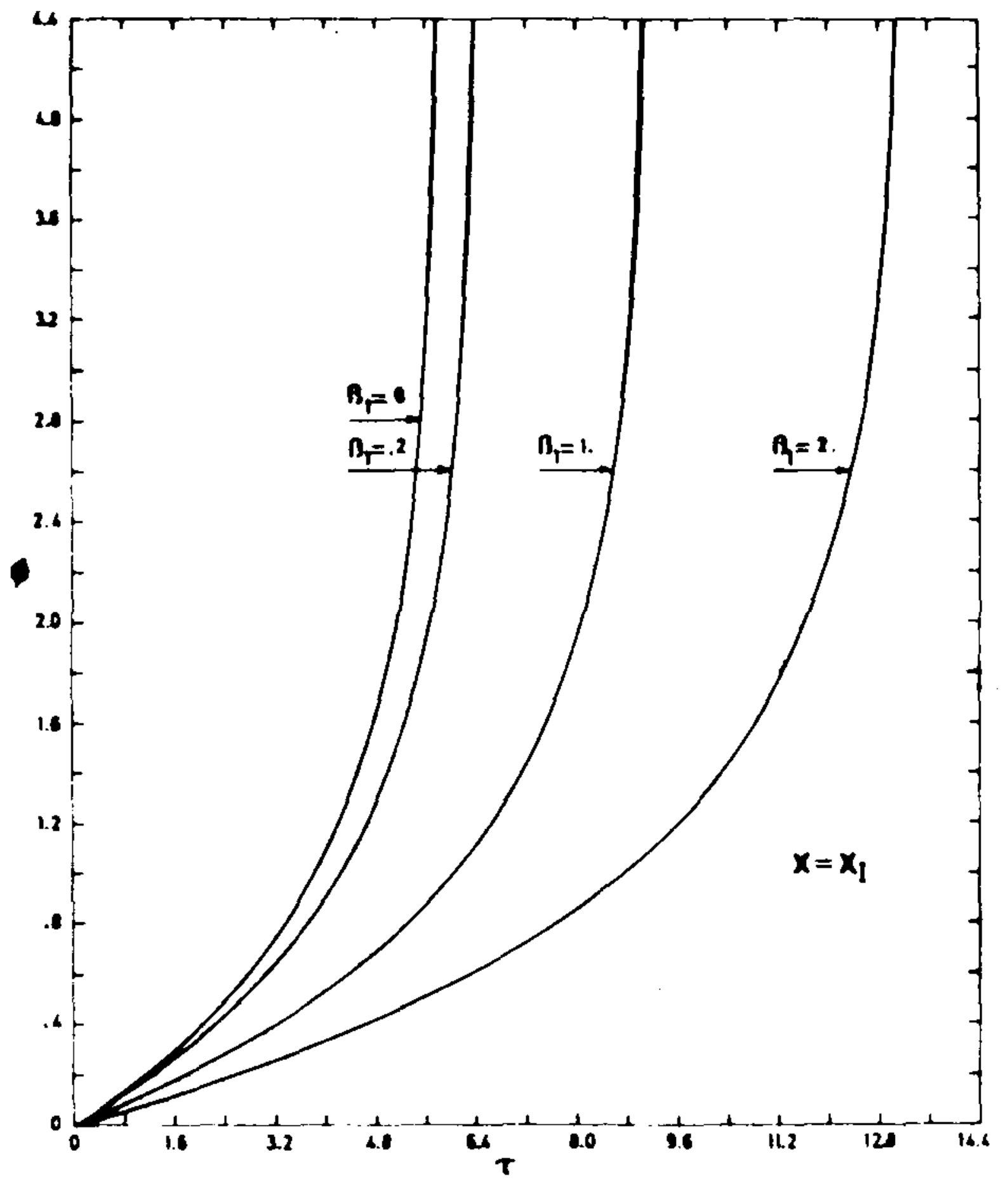

FIGURE 6 Non-dimensional temperature increment at $x=x_{I}$, showing thermal runaway at $\tau=\tau_{I}$. 


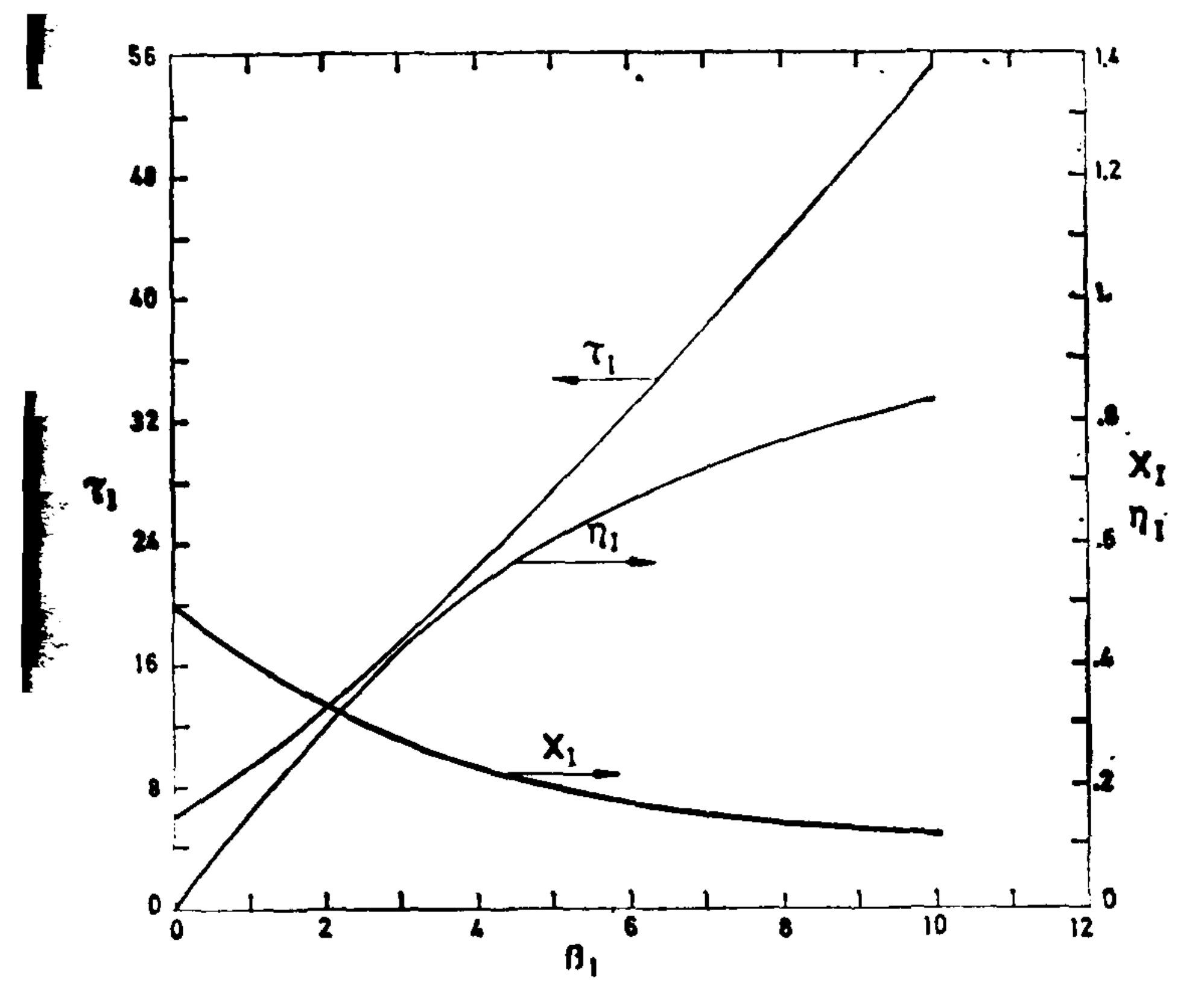

FIGURE 7 Ignition location and ignition time as functions of $\beta_{1}=\left(T_{a} / T_{10^{2}}\right)\left(T_{10}-T_{20}\right)$.

$\eta$ approach $\tau_{I}$ and $\eta_{I}$ but grows rapidly until either $y_{1}$ or $y_{2}$ become very small.

\section{Ignition for Different Initial Temperatures of the Reactants}

When $\beta_{1}$ becomes large, the $\eta_{I}$ of ignition moves toward the region of higher initial temperatures. The Arrhenius exponent causes the heat release in the ignition stage to be negligible in regions where $\epsilon^{-1}\left(T_{f} / T_{10}-1\right)=-\beta_{1} x$ is a large negative number; the chemical reaction is therefore confined to the region where $\beta_{1} x=\xi$, the appropriate variable for the description of the reaction region, is of order unity. For the analysis of the reaction region in the ignition stage we first write the energy equation (31) in terms of the variable $x=(1-\operatorname{erf} \eta) / 2$ so that we obtain the equation

$$
\begin{gathered}
\phi_{\tau}-\frac{\exp \left(-2 \eta^{2}\right)}{4 \pi \tau} \phi_{x x}=\left[1-\epsilon \beta_{1} x+\epsilon \phi\right]^{-(a+b)} \\
\times y_{1}^{a} y_{2}^{b} \exp \frac{\phi-\beta_{1} x}{1-\epsilon \beta_{1} x+\epsilon \phi}
\end{gathered}
$$

Notice that the second term of this equation combines the convective and diffusive terms of Eq. (18). We shall show that this equation in the reaction region simplifies in first approximation to the form given by Eq. (38), representing a quasisteady balance of the diffusive-convective term and the chemical production term, because

i) The chemical production term takes for small $\epsilon$ and large $\beta_{1}$ the simplified form

$$
y_{2}^{b} \exp \left(\phi-\beta_{1} x\right)=\beta_{1}^{-b}(\xi-\beta \phi)^{b} \exp (\phi-\xi)
$$

ii) The factor $\exp \left(-2 \eta^{2}\right)$ in the diffusive-convective term can be replaced for small values of $x$, corresponding to $\beta_{1} x$ of order unity, by its asymptotic form

$$
\exp \left(-2 \eta^{2}\right) \doteq 4 \pi x^{2} \eta^{2}
$$

where the factor $\eta^{2}$ in the right hand side can be replaced by a constant large number $\eta_{1}^{2}$, given below by Eq. (35), with relative errors of order $1 / \eta_{1}{ }^{2}$. To show this, we replace the factor $x^{2}$ in the above equation by $\xi^{2} / \beta_{1}{ }^{2}$ and take logarithms; then, we obtain a relation giving a constant value $\eta_{1}$ for $\eta$, namely,

$$
\eta_{1}^{2}=\ln \left(\beta_{1} / 2 \sqrt{ } \pi\right)-\ln \eta_{1}
$$

if a term, $-\ln \xi$, is neglected in the right hand side. In the reaction zone, where $\xi$ is of order unity, the relation between $\xi$ and $\eta$ is simplified to

$$
\eta=\eta_{1}-\left(1 / 2 \eta_{1}\right) \ln \xi
$$

showing that the reaction zone is located around $z=\eta_{1} 2 \sqrt{ } \tau$ with a thickness of order $V^{\prime}(\tau) / \eta_{1}$.

The convective-diffusive term is thus seen to be of order $\eta_{1}^{2} / \tau$. If we want $\eta_{1}^{2} / \tau$ to be of the order, $\beta_{1}-b$, of the reaction term, the characteristic time for the ignition stage must be of order $\beta_{1}{ }^{b} \eta_{1}{ }^{2}$. As a consequence, for the analysis of the reaction layer in the ignition stage, we shall use the variables

$$
\xi=\beta_{1} x \quad \text { and } \quad \tau_{1}=\tau / \beta_{1}^{b} \eta_{1}^{2}
$$

When Eq. (34) is written in terms of these variables, we find that it reduces, if we neglect terms of order $1 / \eta_{1}{ }^{2}$ compared with those retained, to the ordinary differential equation,

$$
-\xi^{2} \tau_{1}{ }^{-1} \phi_{\xi \xi}=(\xi-\beta \phi)^{b} \exp (\phi-\xi),
$$

where $\tau_{1}$ acts as a parameter together with $\beta, \uparrow$

$$
\beta=c_{p}\left(T_{10}-T_{20}\right) / Q Y_{20}
$$

+ The parameter $\beta$ measures the initial difference in temperature of oxidizer and fuel relative to the increment in temperature due to the reaction. According to Eq. (25), the adiabatic flame temperature $T_{e}$ is larger than $T_{10}$, if $\beta<1$, and vice versa if $\beta>1$. 
Notice that the left hand side of Eq. (38) is obtained from Eq. (18) if the unsteady term is neglected, the factor $\eta$ in the convective term is replaced by $\eta_{1}$, and Eq. (36) is used as the appropriate simplified relation between $\eta$ and $\xi$ in the reaction zone.

Eq. (38) will be integrated with the boundary conditions $\phi(0)=0, \phi_{\xi}(\infty)=0$. From the results of this integration we shall obtain $\phi(\infty)=\phi_{\infty}\left(\tau_{1}\right)$.

The boundary condition $\phi_{\varepsilon}(\infty)=0$ for the solution in the reaction zone is obtained from the matching conditions with the solution of the heat conduction equation in the much thicker chemically frozen region, $\eta / \eta_{1}<1$. In this region, where $\eta \sim \eta 1$, Eq. (18) simplifies to

$$
\frac{\partial \phi}{\partial \tau_{1}}-\frac{\eta}{2 \tau_{1}} \frac{\partial \phi}{\partial \eta}=0
$$

because the diffusive term is of the order $1 / r_{1}{ }^{2}$ compared with the terms retained, and the chemical production term is negligible.

The general solution of Eq. (40) is $\phi=f\left(\eta^{2} \tau_{1}\right)$, or equivalently $\phi=f_{1}(z)$, where the function $f$ is to be determined from the matching conditions, for $\eta \rightarrow \eta 1$, with the solution for the reaction region. Thus, the temperature increment due to the chemical reaction in the region where $1 \ll \eta<\eta_{1}$ is given by

$$
\phi=\phi_{\infty}\left(\tau_{1} \eta^{2} / n_{1}{ }^{2}\right)
$$

In the region where $\eta \sim 1$, or negative, $\phi$ is zero in first approximation.

Figure 8 shows $\phi(\xi)$ for $b=1$, several values of $\tau_{1}$, and three representative values of $\beta$, as obtained by numerical integration of Eq. (38). Figure 9 shows $\phi_{\infty}$ in terms of $\tau_{1}$ for several values of $\beta$. From the results we notice that if $\beta<1$, there are two values $\dagger$ of $\phi_{\infty}$ for all times lower than a maximum value $\tau_{I 1}$, which is a function of $\beta$, and no solutions for $\tau_{1}<\tau_{I 1} ; \tau_{I 1}$ becomes infinite when $\beta$ approaches 1 . For $\beta>1$, the relation $\phi_{\infty}\left(\tau_{1}\right)$ is single-valued. When $\beta<1$ and $\tau$ approaches $\tau_{I 1}$, $\partial \phi_{\infty} / \partial \tau_{1}$ becomes infinite and the quasi-steady equation (38) ceases to be valid because the time derivative can no longer be neglected in Eq. (34). We may then expect that the solution of the complete energy equation will show that the temperature will keep increasing very rapidly so that local

† The model equation (38) does not provide an upper bound for the temperature increment $\phi$, and consequently no triple valued solutions are found for $\phi_{\infty}\left(\tau_{1}\right)$ as sometimes occurs in other combustion problems.
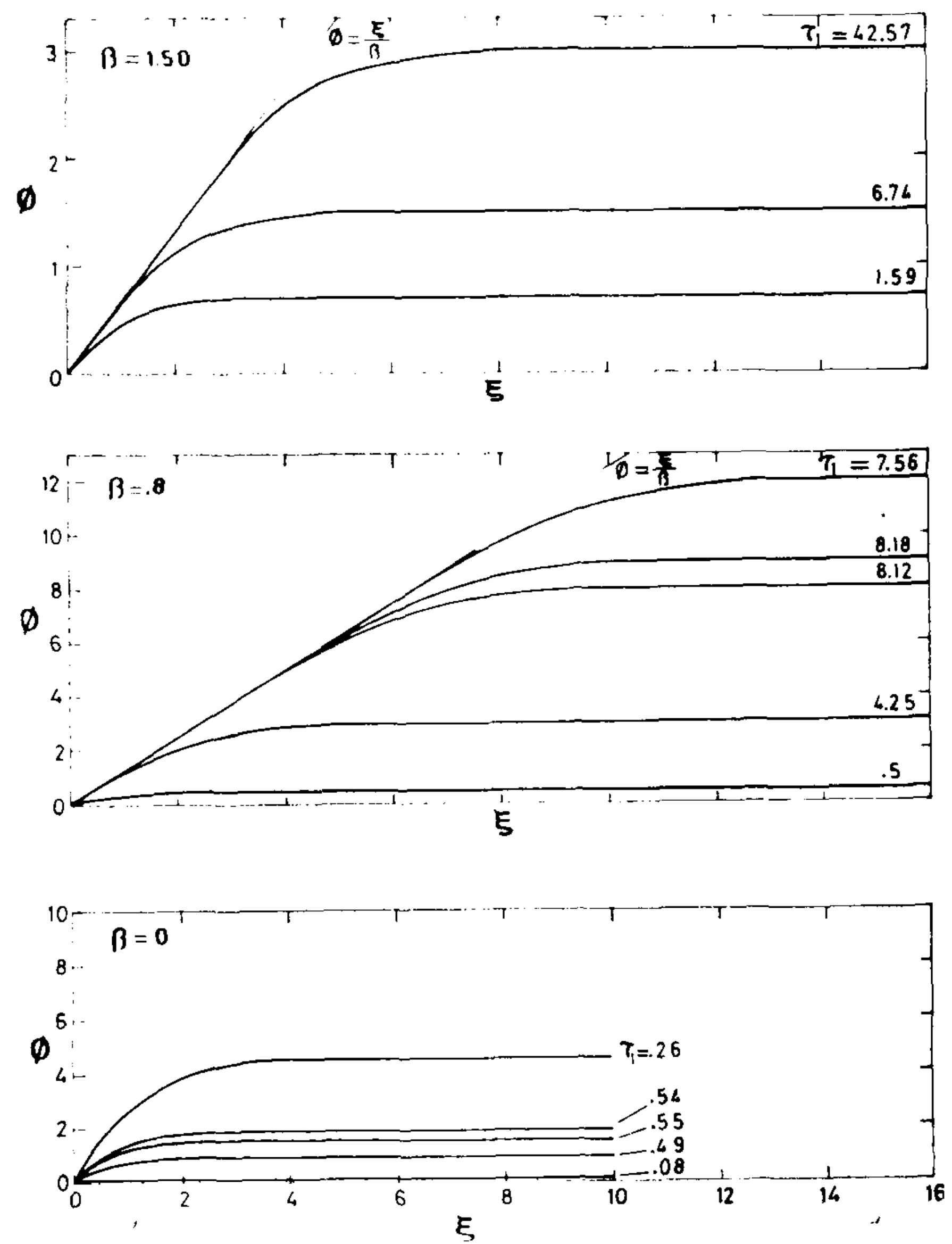

FIGURE 8 Temperature increment due to chemical reaction during the ignition stage, for initial differences in temperature of the order of the adiabatic flame temperature, as predicted by the quasi-steady analysis for the reaction layer.

equilibrium, with $y_{2}=0$, will be reached shortly after $\tau=\tau_{I 1}$. The non-existence of solutions of the quasi-steady Eq. (38) for $\beta<1$ at times larger than $\tau_{I 1}$, and the rapid increase in chemical-heat release at times close to $\tau_{I 1}$ may be used as indicative of ignition, and $\tau_{I 1}$ may be considered as the ignition time.

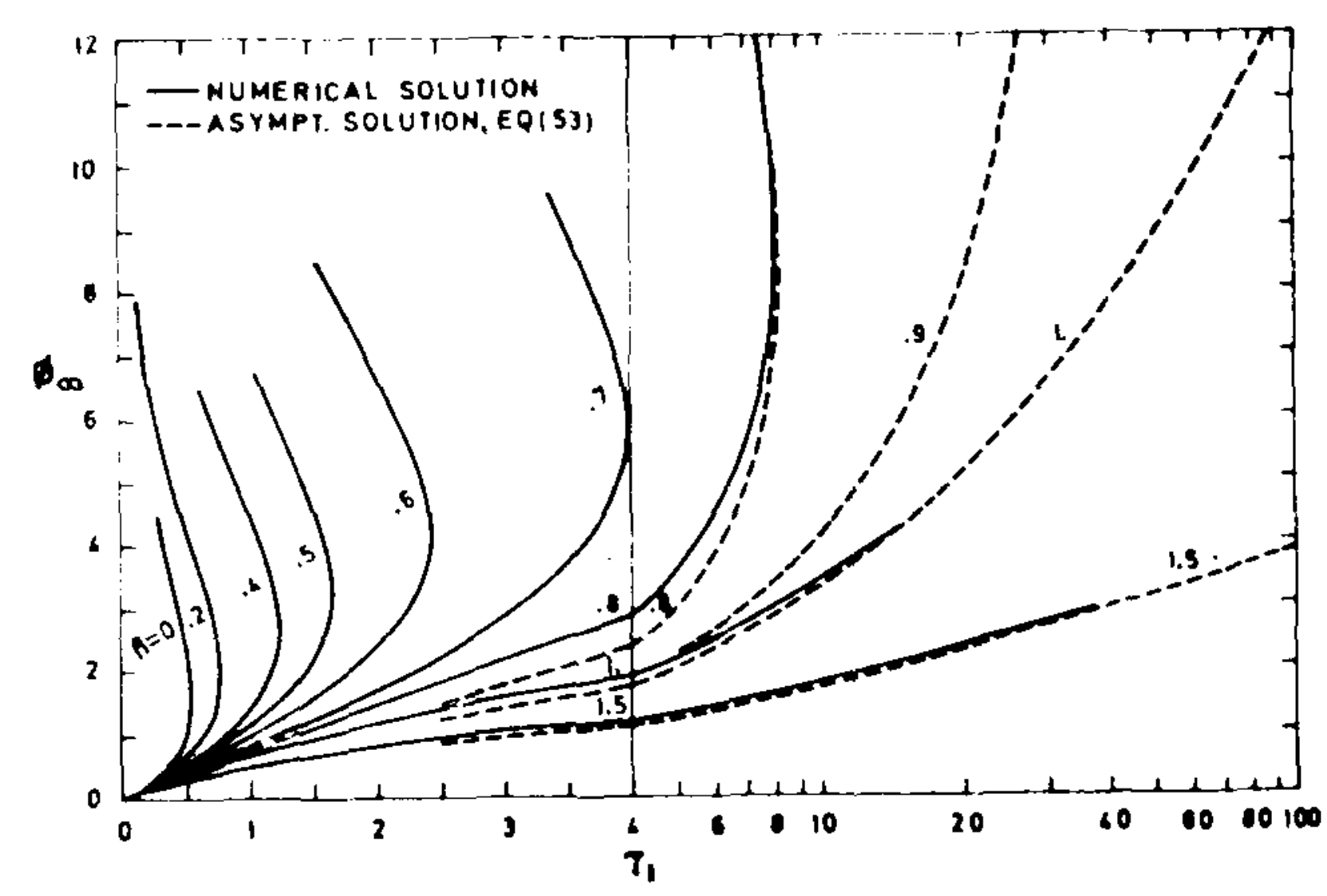

FIGURE 9 The maximum increment in temperature resulting from the quasi-steady analysis of the ignition stage. For $\beta<1$ the relations $\phi_{\infty}\left(\tau_{1}\right)$ are double branched, and only the lower one has physical significance. 
For times of the order of $\tau_{I 1}$, which are large when $1-\beta$ is small, and for large $\tau_{1}$ when $\beta>1$, the numerical solutions in Figure 8 show the existence of a region where chemical equilibrium $(\phi=\xi / \beta)$ has already been reached. This region is separated from a region of frozen flow $\left(\phi=\phi_{\infty}\right)$ by a transition reaction region located around $\xi=\beta \phi_{\infty}$ where $\phi_{\infty}$ is large. This transition region is thin compared with the equilibrium region, at smaller $\xi$, or the chemically frozen region, at larger $\xi$. We may use the fact that $\phi_{\infty}$, in these cases, is a large number, to obtain analytical expressions for $\phi_{\infty}\left(\tau_{1}, \beta\right)$ and $\tau_{I 1}(\beta)$. To do this we shall pose our problem so as to find the solution of Eq. (38) and the value of $\tau_{1}$ for which $\phi(\infty)$ is a given large number $\phi_{\infty}$. We shall only consider here the case $b=1$.

Anticipating that for large $\phi_{\infty}$ the chemical reaction will only be important at values of $\xi$ close to the large value $\beta \phi_{\infty}$, and that there the deviations of $\phi$ from its local equilibrium value $\xi / \beta$ will be of order unity, we shall write Eq. (38) in terms of the dependent variable $\gamma$, proportional to the fuel mass fraction,

$$
\gamma=\xi / \beta-\phi
$$

and the independent variable $\zeta$,

$$
\zeta=\left[\xi-\beta\left(\phi_{\infty}+p_{0}\right)\right] / \beta \text {. }
$$

The stretching factor $\beta$, and the additional translation $\beta p_{0}$ (which we anticipate to be of order unity) are chosen so as to obtain a certain normalized equation, [Eq. (47)] and boundary conditions [Eq. (48)], in the limit $\phi_{\infty} \rightarrow \infty$.

When Eq. (38) is written in terms of $\gamma$ and $\zeta$, it takes the form,

$\left[1+\left(\zeta+p_{0}\right) / \phi_{\infty}\right]^{2} d^{2} \gamma / d \zeta^{2}$

where

$$
=\Lambda \gamma \exp [-\gamma+(1-\beta) \zeta]
$$

$$
\Lambda=\tau_{1} \beta \phi_{\infty}{ }^{-2} \exp \left[(1-\beta)\left(\phi_{\infty}+p_{0}\right)\right],
$$

Equation (44) must be solved with the boundary conditions

$$
\begin{gathered}
\gamma=0 \text { at } \zeta=-\left(\phi_{\infty}+p_{0}\right) \\
\gamma-\zeta=p_{0} \text { for } \zeta \rightarrow \infty
\end{gathered}
$$

These boundary conditions correspond to the conditions $\phi=0$ at $\xi=0$, and $\phi=\phi_{\infty}$ at $\xi \rightarrow \infty$, respectively.

It is not difficult to show that the factor $\Lambda$ in Eq. (44) may be chosen arbitrarily, and then
$p_{0}\left(\Lambda, \beta, \phi_{\infty}\right)$ will be obtained as an eigenvalue when solving Eq. (44) with the boundary conditions (46). Because these equations have no solution in the limit $\phi_{\infty} \rightarrow \infty$, for $\beta=1$ unless $\Lambda=1 / 2$, we shall choose $\Lambda=1 / 2$ in Eq. (44).

The problem of solving Eq. (44) for large $\phi_{\infty}$ turns out to be a singular perturbation problem. There is an inner reaction layer where the appropriate independent variable is $\zeta$. In addition, there is an outer layer to the left, where the appropriate independent variable is $\xi$, with the chemical reaction frozen to orders exponentially small in $\phi_{\infty}$ if $\beta<1$, or in equilibrium if $\beta>1 \dagger$. To the right of the inner reaction layer, the chemical reaction is frozen to all algebraic orders in $\phi_{\infty}^{-1}$, so that $\gamma-\zeta=p_{0}$; the eigenvalue $p_{0}$ admits an expansion in powers of $\phi_{\infty}{ }^{-1}$ beginning with a term of order unity.

The first approximation for $\gamma$, within the reaction layer, can be shown to be given by the equation,

$$
2 d^{2} \gamma / d \zeta^{2}=\gamma \exp (-\gamma+m \zeta)
$$

where $m=1-\beta$, to be solved with the boundary conditions

$$
\begin{array}{r}
\gamma_{\zeta}=0 \text { for } \zeta \rightarrow-\infty, \text { and } \\
\gamma_{\zeta}=1 \text { for } \zeta \rightarrow \infty .
\end{array}
$$

This equation has been integrated numerically to obtain $\gamma(\zeta, m)$, and, as part of the solution, we then obtain the first approximation for $p_{0}, p_{0}=$ $(\gamma-\zeta)_{\infty}$, which is a function only of $m$ shown in Figure 10; also shown in Figure 10 is $\gamma_{-\infty}$, which is identically zero for $m \leqslant 0$. There are no solutions for $m>1 / 2$, or $\beta \leqslant 1 / 2$.

The second approximation for $\phi$ in the equilibrium or frozen region for $\xi<\beta \phi_{\infty}$ is given by $\phi=\xi / \beta-\xi \gamma_{-\infty} / \beta \phi_{\infty}$.

Asymptotic methods may also be used (Liñán, 1973), to obtain analytical expressions for $(\gamma-\zeta)_{\infty}$ and $\gamma_{-\infty}$ in terms of $m$, for values of $m$ close to $1 / 2$, 0 , or large negative $m$. By taking into account these asymptotic expressions, the following correlations, with relative errors of the order of 2 per-

\footnotetext{
$\dagger$ It should be noticed, however, that because of the factor $\xi^{2}$ in Eq. (38), chemical equilibrium will always exist for all values of $\tau_{1}$, in the region where $\xi^{2}$ is small enough to make the reaction term dominant if $\xi-\beta \phi$ does not tend to zero with $\xi$ faster than $\xi$. Even though the chemical reaction is frozen for $\xi<\phi_{\infty}$ and $\beta<1$, equilibrium must exist there in the first approximation, if matching with the reaction layer is to be possible.
} 


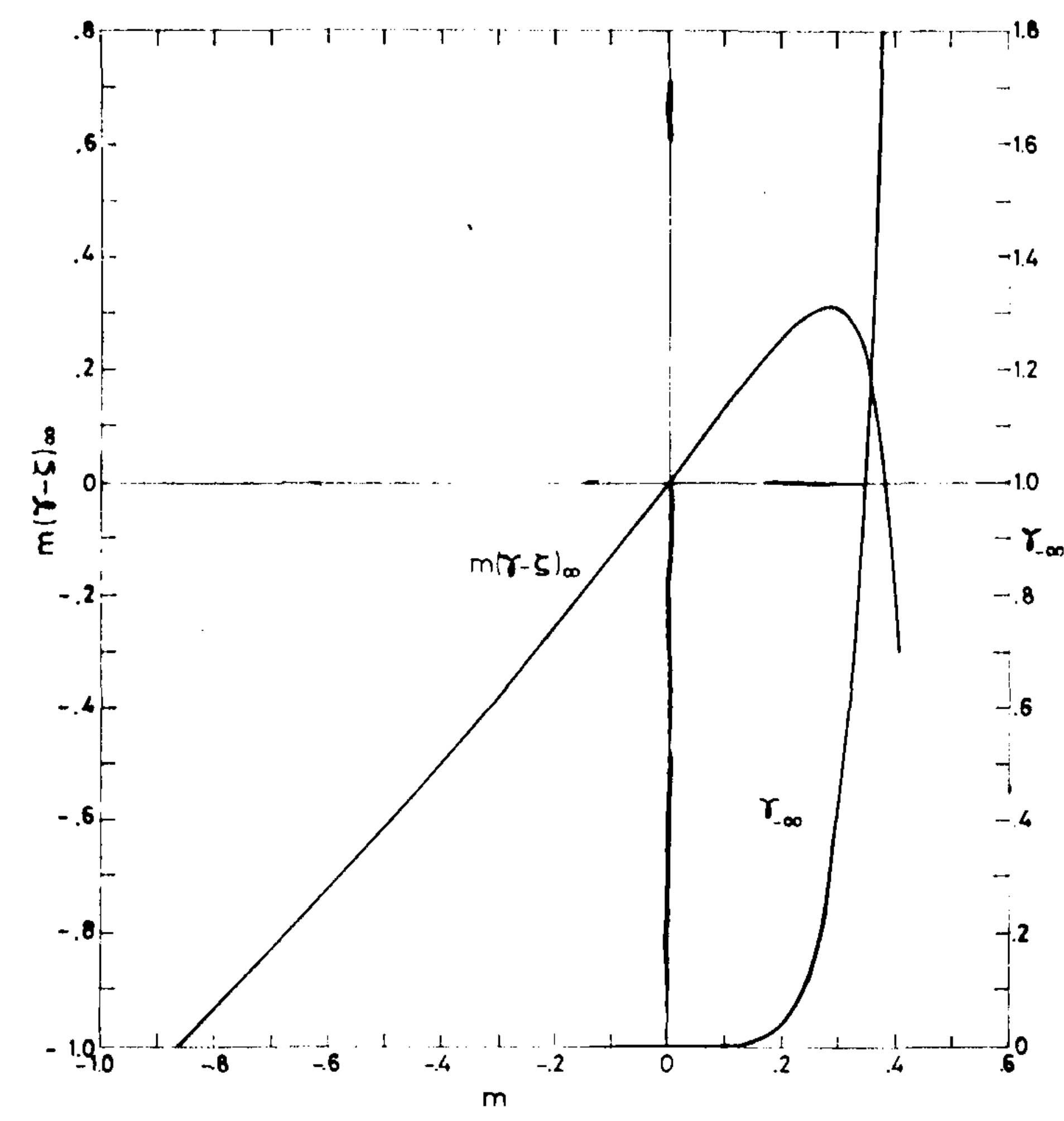

FIGURE 10 Asymptotic values resulting from the Eqs. (47) and (48), describing the reaction zone structure of premixed flames.

cent or lower, were found for $(\gamma-\zeta)_{\infty}$ as a function of $m$.

$$
\begin{aligned}
m(\gamma-\zeta)_{\infty}=1.344 m & -4 m^{2}(1-m) /(1-2 m) \\
& +3 m^{3}-\ln \left(1-4 m^{2}\right)
\end{aligned}
$$

for $m>-0.2$, and

$$
m(\gamma-\zeta)_{\infty}=-\ln \left(0.6307 m^{2}-1.344 m+1\right)
$$

for negative values of $m$.

With $p_{0}$ known as a function of $\beta$, Eq. (45), with $\Lambda=1 / 2$, provides a first approximation to the relation between $\tau_{1}, \phi_{\infty}$ and $\beta$, as

$$
\tau_{1}=(1 / 2 \beta) \phi_{\infty}^{2} \exp \left[(\beta-1)\left(\phi_{\infty}+p_{0}\right)\right]
$$

The relation $\phi_{\alpha}\left(\tau_{1}\right)$, resulting from Eq. (50), is single-valued only for $\beta>1$. For $\beta<1, \phi_{\infty}\left(\tau_{1}\right)$ has two branches; the lower branch is not a good approximation to the solution of Eq. (38), unless $1-\beta$ is moderately small, because the values of $\phi_{\infty}$ are not large enough to make the asymptotic treatment applicable.

If $(1-\beta)$ is small, the ignition time, $\tau_{I 1}$, and the flame location at that time, $\xi_{I}=\beta \phi_{\infty}$, are given by

$$
\tau_{I 1}=2 e^{-2} /(1-\beta)^{2}, \quad \xi_{I}=2 \beta /(1-\beta)
$$

corresponding to the value $\phi_{\infty I}=2 /(1-\beta)$ of $\phi_{\infty}$ that makes $\tau_{1}$ the maximum in Eq. (50).

For smaller values of $\beta, \tau_{I 1}(\beta)$ must be obtained from the numerical solution of Eq. (38). A good analytical correlation of these numerical results is given by

$$
\tau_{I 1}=2 e^{-2}(2-\beta) /(1-\beta)^{2}
$$

It is easy to obtain a second approximation for the value of $\tau_{1}$ and $\tau_{I 1}$ for large values of $\phi_{\infty}$. In Eq. (44) the first term in brackets is expanded for large $\phi_{\infty}$ and the resulting equation is manipulated so as to obtain an equation similar to (47). The second approximation to the value of $\tau_{1}$ is:

$$
\begin{aligned}
\tau_{1}= & \frac{1}{2 \beta} \phi_{\infty}^{2} \exp \left[(\beta-1)\left(\phi_{\infty}+p_{0}\right)\right] \\
& \times\left[1-\frac{2}{\phi_{\infty}} \frac{\partial(1-\beta) p_{0}}{\partial \beta}\right]
\end{aligned}
$$

where $p_{0}$, as a function of $\beta$, is given by Eq. (49).

Equation (53) can be used to obtain a second approximation to the value of $\tau_{I 1}$, for small values of $(1-\beta)$. The resulting expression is Eq. (52), which turns out to be a good correlation of the numerical results. (For example, for $\beta=0$, Eq. (52) gives $\tau_{I 1}=0.541$ while the numerical results give $\tau_{I 1}=0.547$ ).

The relation (52) gives explicitly the ignition time, for $\beta>1$ and $b=1$, in terms of the physical and chemical parameters of the system, if Eqs. (13), (35) and (37) are taken into account. Because for $\beta \rightarrow 1, \xi$ is large, $\xi \simeq 2 /(1-\beta)$, in the reaction region, the $\eta_{1}$ appearing in Eq. (37) for $\tau_{1}$, should be redefined by replacing $\beta_{1}$ in Eq. (35) by $\beta_{1}(1-\beta) / 2$.

It should be noticed that the ignition time does not depend on the transport coefficients. It depends only on the initial thermodynamic state of the reactants and the chemical kinetic parameters of the reaction.

It is clear that the analysis may be used to predict the ignition time when a premixed reacting mixture is suddenly put in contact with a hot inert gas, or the ignition delay length of a premixed reactant stream flowing parallel to a hot inert stream. With minor modifications this analysis may also be used to predict the ignition time in a wide variety of problems. Some examples are, the ignition of monopropellant and bipropellant droplets, and gas phase ignition of a solid propellant in contact with a hot gas. 


\section{IV}

We have found in the previous section that, if $\beta<1$, duc to the large sensitivity of the reaction rate with temperature, the chemical heat release causes the temperature to rise precipitously at a certain ignition time, at a certain place somewhere in the mixing layer. It is clear that the local rise in temperature must cease when one of the reactants is completely consumed at the ignition spot; there, the final temperature will be given by Eq. (15) with $y_{1}=0$ or by Eq. (16) with $y_{2}=0$, depending on which of the reactants is in defect.

These facts were well demonstrated for small values of $1-\beta$; for which it was found that, well before ignition occurred, a thin reaction layer, or flame, was separating a region of frozen flow from a region of near-equilibrium flow. The reaction was found to move in time toward regions of increasing initial fuel concentration and thereby of increasing final equilibrium temperature; for this reason the flame propagation velocity keeps increasing with time.

The heat released at the reaction layer is conducted both to the frozen region, ahead of the layer, and to the near-equilibrium region, behind. Because during most of the ignition stage the thickness of the near-equilibrium region is small compared with the thickness of the, inert, unsteady transport layer, the heat loss toward this layer from the reaction layer was neglected, resulting in the boundary condition $\phi_{\xi}(\infty)=0$ for Eq. (38). As a consequence of this approximation the propagation velocity of the reaction layer became infinite at $\tau_{1}=\tau_{I 1}$

However, when $\tau_{1}$ approaches $\tau_{I 1}$, the propagation velocity becomes so large that heat conduction ahead of the flame cannot take place so rapidly as to make the thickness of the unsteady transport layer large compared with the thickness of the near-equilibrium layer. Therefore, the boundary condition $\phi_{\xi}(\infty)=0$ should no longer be used. The fact that, for $\tau$, close to $\tau_{I 1}$, the unsteady transport layer is then thin compared with the mixing layer will be used below in order to simplify the Eq. (40), which describes how the chemical heat relaese is conducted and stored ahead of the reaction layer.

For $\beta>1$ and large $\tau_{1}$, we also found a thin reaction region separating a region of equilibrium flow from a region of frozen flow. In this case, however, the flame moves in time toward regions of lower final equilibrium temperature; thereby, its propa- gation velocity becomes so small that the characteristic time for motion of the flame across the mixing layer is large compared with the time necessary for the heat released at the flame to be conducted across the mixing layer. In this case the time derivative in Eq. (40) turns out to be negligible; and the temperature in the transport layer will be given by $\phi=(1-x) \phi_{\infty}$, as long as the equilibrium layer is thin compared with the mixing layer. Because for sufficiently large values of $\tau_{1}$ this is not the case, Eq. (38) cannot then be used in the equilibrium and reaction layers.

\section{Flame Propagation for $\mathrm{T}_{10}-\mathrm{T}_{20}>\mathrm{QY}_{20} / \mathrm{c}_{\mathrm{p}}$}

We shall now analyze the flame propagation process for $\beta>1$ and large values of $\tau$. Guided by the results obtained in the previous section, we anticipate that a thin reaction layer set around $x=x_{p}(\tau)<x_{e}$, separates a region of frozen flow from a region of equilibrium flow, at $x<x_{p}$ (where $x$ is not so close to $x_{p}$ as to be in the reaction region).

In the equilibrium region, according to Eqs. (15) and (16),

and

$$
T-T_{10}=-x\left(T_{10}-T_{20}-Q Y_{20} / c_{p}\right)
$$

$$
y_{2}=0, \quad y_{1}=1-x\left(1+\nu Y_{20} / Y_{10}\right)
$$

In the frozen layer, ahead of the reaction zone, the temperature distribution obeys the heat conduction Eq. (18) (without the chemical source term), with a moving boundary condition, $T=T_{p}(\tau)$ at $x=x_{p}(\tau)$, originating from the matching conditions with the solution for the reaction layer. $T_{p}(\tau)$ is the first approximation for the temperature in the reaction zone.

Because the changes in temperature and concentration across the reaction zone, as well as the reaction zone thickness, will be found to be small, of order $T_{p} / T_{a}$ (compared with the transport zone), in the first approximation we may patch at $x=x_{p}(\tau)$ the solution, Eq. (54), for the equilibrium region to the solution in the transport region.

The movement of the reaction layer is so slow (that is $x_{p}(\tau)$ and $T_{p}(\tau)$ are such slow varying functions of time) that, as indicated above, the time derivative term in Eq. (18) turns out to be also negligible. The temperature for $x>x_{p}$ ( $x$ not too close to $x_{p}$ ) is then given by the local similarity solution,

$$
T=T_{20}+\left(T_{p}-T_{20}\right)(1-x) /\left(1-x_{p}\right)
$$


where $T_{p}$ is the value of $T$ given by Eq. (54) at $x=x_{p}$,

$$
T_{p}=T_{10}-x_{p}\left(T_{10}-T_{20}-Q Y_{20} / c_{p}\right)
$$

In order to determine now $x_{p}(\tau)$, or $T_{p}(\tau)$, and the temperature distribution in the reaction layer, we pose the inverse problem of calculating the value of $\tau$ which causes the temperature to take the value given by Eq. (54) in the equilibrium region for $x<x_{p}$ (because there the characteristic chemical time will be exponentially large for large values of $\left.T_{a} / T_{p}\right)$, and the value given by Eq. (56) in the frozen region for $x>x_{p}$ (where the chemical time will be exponentially large).

To obtain the temperature distribution in the reaction layer around $x=x_{p}$ we will follow the procedure followed in the previous section to obtain Eq. (47). We shall give the details of the analysis only for the case $b=1$.

We should write Eq. (14) in terms of the dependent variable $\gamma=y_{2} Q Y_{20} T_{a} / c_{p} T_{p}{ }^{2}$, or equivalently,

$$
\begin{aligned}
\gamma=-T_{a}\left(T-T_{p}\right) / T_{p}{ }^{2}- & \left(x-x_{p}\right) \\
& \times\left(T_{10}-T_{20}-Q Y_{20} / c_{p}\right) T_{a} / T_{p}{ }^{2}
\end{aligned}
$$

and the independent variable

$$
\zeta=-p_{0}+\left(Q Y_{20} T_{a} / c_{p} T_{p}^{2}\right)\left(x-x_{p}\right) /\left(1-x_{p}\right)
$$

which are chosen, together with $p_{0}$, so that for large values of $T_{a} / T_{p}$, we obtain Eqs. (47) and (48) to determine the first approximation for $\gamma(\zeta)$. The resulting value for $m$ in Eq. (47) is negative, and given by,

$$
m=(1-\beta)\left(1-x_{p}\right)
$$

The translation $p_{0}$ in Eq. (59) is given by the relation

$$
\begin{gathered}
\frac{8 \pi \tau y_{1 p}\left(1-x_{p}\right)^{2}}{\exp \left(-2 \eta_{p}^{2}\right)}\left(\frac{c_{p} T_{p}^{2}}{Q Y_{20} T_{a}}\right)^{3}\left(\frac{T_{10}}{T_{p}}\right)^{3+a} \\
\quad \times \exp \left[\frac{T_{a}}{T_{10}}-\frac{T_{a}}{T_{p}}+m p_{0}\right]=1
\end{gathered}
$$

where

$$
y_{1 p}=1-x_{p}\left(1+\nu Y_{20} / Y_{10}\right)
$$

and

$$
1-\operatorname{erf} \eta_{p}=2 x_{p}
$$

We should notice that Eq. (47), which describes the reaction zone structure, represents a balance between the chemical production term and conduction, or diffusion, normal to the flame. When calculating the reaction term, a constant value is taken for the oxidizer concentration $y_{1 p}$ in the reaction layer, and the Arrhenius exponent is linearized around the value $T_{p}$ of $T$.
The stretching factor in $\zeta$, which results in the expression (60) for $m$, was chosen so as to obtain the boundary condition $\gamma_{\zeta}=1$ for $\zeta \rightarrow \infty$ from the matching conditions with the outer solution Eq. (56). From these conditions we also obtain,

$$
(\gamma-\zeta)_{\infty}=p_{0}
$$

which when substituted in Eq. (61) yields, if Eq. (49b) is taken into account, an approximate relation giving $\tau$ in terms of $T_{p}$ with relative errors of order $T_{p} / T_{a}$.

When $T_{p}$ approaches $T_{e}$, the resulting value of $\tau$ tends to infinity, because $y_{1 p} \rightarrow 0$. However, the preceding analysis is then no longer applicable, and should be replaced by an analysis in which both $y_{1}$ and $y_{2}$ should be considered small, of the order $T_{e} / T_{a}$, in the reaction zone. For these large values of time, the region $x<x_{e}$ is in equilibrium (with $y_{2}=0$ ), while the region $x \geqslant x_{e}$ is in nearequilibrium (with $y_{1} \ll 1$ ). The detailed analysis of this stage, which corresponds to a diffusion controlled mode of combustion will be given in Section V.

\section{Flame Propagation for $\mathrm{T}_{10}-\mathrm{T}_{20}<\mathrm{QY}_{20} / \mathrm{c}_{\mathrm{p}}$}

If $\beta<1$, the thin reaction zone, that sets the flow in near-equilibrium, once it has been established at $\tau_{1}$ close to $\tau_{I 1}$, moves across the mixing layer in an interval of time which is very short compared with the ignition time.

Because of this fast motion of the reaction layer, the heat released by the chemical reaction will only reach a transport layer, which is thick compared with the reaction layer but thin compared with the mixing layer at the time of ignition. The transport layer precedes the reaction layer in its motion across the mixing layer. Ahead of the transport layer the temperature and concentration take the frozen flow values, Eq. (10), if we neglect terms of order $T_{10} / T_{a}$ due to the effect of the chemical reaction during the ignition stage.

The reaction layer together with the transport layer form a thin transition layer, through which the temperature and concentrations jump from its frozen flow values just ahead to values very close to equilibrium just behind. This transition layer is a classical deflagration. An analysis of the deflagration propagation for large activation energies was carried out by Zeldovich and Frank-Kamenetsky (1938). Later, this analysis was refined and carried out to higher orders, by means of singular 
perturbation methods, by Jain and Kumar (1969) and Bush and Fendell (1970).

If the activation energy is sufficiently large, the chemical reaction takes place only in a thin reaction zone, which we may then place around a certain $x_{p}(\tau)$, where the temperature is very close to the equilibrium value $T_{p}$ at $x=x_{p}$.

The reaction layer leaves behind a region in near-equilibrium, where Eqs. (54) and (55) will hold in the first approximation, if $x_{p}<x_{e}$. The reaction layer, after crossing the surface $x=x_{e}$, leaves behind, in addition to the region, $x<x_{e}$, where as before $y_{2}=0$ in the first approximation, a second region, $x_{p}>x>x_{e}$, where $y_{2} \neq 0$ but $y_{1}=0$. These two regions are separated by a thin reaction zone, around $x=x_{e}$, which is a typical thin diffusion flame to be analyzed in Section V.

In the transport layer preceding the reaction layer, where the reaction term turns out to be zero to all algebraic orders in $T_{p} / T_{a}$, the temperature is given by the heat conduction equation

$$
\frac{\partial T}{\delta \tau}-\frac{\exp \left(-2 \eta^{2}\right)}{4 \pi \tau} \frac{\partial^{2} T}{\partial x^{2}}=0
$$

which we shall write in terms of the variables $\tau$ and $\zeta_{1}=x-x_{p}(\tau)$, anticipating that $\zeta_{1} / x_{p} \ll 1$ in the transport zone. We thus obtain, for large $T_{a} / T_{p}$, in the first approximation

$$
\dot{x}_{p} \frac{\partial T}{\partial \zeta_{1}}+\left(\frac{x_{p}^{2}}{\tau E_{p}}\right) \frac{\partial^{2} T}{\partial \zeta_{1}^{2}}=0
$$

where $\dot{x}_{p}=d x_{p} / d \tau$, and $E_{p}=4 \pi x_{p}{ }^{2} \exp \left(2 \eta_{p}{ }^{2}\right)$.

We have not included in Eq. (66) a term ( $\partial T /$ $\partial \tau)_{\zeta 1}$, because it turns out to be negligible compared with the two remaining terms in the equation.

Equation (66) has the solution

$$
\begin{aligned}
T=T_{10}+\left(T_{20}-T_{10}\right) x_{p}+\left(x_{p} Q Y_{20} / c_{p}\right) \\
\times \exp \left\{-E_{p} \tau x_{p}^{-2} \dot{x}_{p}\left(x-x_{p}\right)\right\}
\end{aligned}
$$

which for large values of $\tau E_{p}\left(x-x_{p}\right) x_{p}{ }^{-2} \dot{x}_{p}$ tends toward the frozen flow solution. In addition, for $x=x_{p}, T$ takes the value $T_{p}=T_{10}+x_{p}\left(Q Y_{20} / c_{p}-\right.$ $\left.T_{10}+T_{20}\right)$; so that the solution, Eq. (67), for the transport layer matches with the one corresponding to the reaction layer, in which the deviations in temperatures from $T_{p}$ are of order $T_{p} / T_{a}$. From Eq. (67) it can be seen that $E_{p} \tau x_{p}{ }^{-1} \dot{x}_{p} \gg 1$ if $x-x_{p}$ $\ll x_{p}$ in the transport layer.

For the analysis of the reaction zone, we introduce, as we did when analyzing the case $\beta>1$, the dependent variable $\gamma=y_{2}\left(T_{a} / T_{p}\right) Q Y_{20} / c_{p} T_{p}$, which is given by Eq. (58), and the independent variable $\zeta$, to be substituted in Eq. (18),

$$
\begin{aligned}
\zeta= & -p_{0}+\left(x-x_{p}\right)\left(T_{a} / T_{p}\right) \\
& \times\left(Q Y_{20} / c_{p} T_{p}\right)\left(E_{p} \tau x_{p}{ }^{-1} \dot{x}_{p}\right)
\end{aligned}
$$

The stretching factor in Eq. (68), and the translation $p_{0}$ are chosen so as to obtain, for $b=1$, Eqs. (47) and (48), when determining the first approximation for $\gamma$ in an asymptotic analysis for large values of $T_{a} / T_{p}$. The matching conditions with the solution, Eq. (67), for the transport layer yield Eq. (64) and the stretching factor in Eq. (68). The parameter $m$ appearing in Eq. (47) is in this case a small number given by,

$$
m=(1-\beta) /\left(E_{p} \tau x_{p}^{-1} \dot{x}_{p}\right)
$$

and therefore $p_{0}=1.344$, as given by Eq. (49a). Taking into account the definition of $p_{0}$ given above, we obtain the relation

$$
\begin{aligned}
\tau \dot{x}_{p}^{2}= & {\left[2 y_{1 p}^{a} E_{p}^{-1}\left(\frac{c_{p} T_{p}^{2}}{Q Y_{20} T_{a}}\right)^{3}\left(\frac{T_{10}}{T_{p}}\right)^{a+3}\right.} \\
& \left.\exp \left(\frac{T_{a}}{T_{10}}-\frac{T_{a}}{T_{p}}\right)\right]
\end{aligned}
$$

where a factor $\exp (1.344 m)$ has not been included because $m \ll 1$.

From Eq. (70) we obtain two values for $\sqrt{ }(\tau) \dot{x}_{p}$ as a function of $x_{p}$, one positive and the other negative. These values correspond to deflagrations moving toward the regions of increasing and decreasing $x$, respectively.

The trajectories of the two flames can be obtained by quadrature, if we add an initial condition

$$
x_{p}=x_{i} \text { at } \tau=\tau_{i}
$$

If we could, artificially, at a certain $\tau=\tau_{i}<\tau_{I}$, raise locally the temperature somewhere in the mixing layer $\left(x=x_{i}\right)$, so that local equilibrium, with $y_{2}=0$, would be very rapidly established there, $x_{i}$ and $\tau_{i}$ would then provide the initial condition, Eq. (71). Two deflagrations, originating at the surface $x=x_{i}$, would then move across the mixing layer to put it in equilibrium. Their trajectories would be obtained by integrating Eq. (70), if $x_{i}<x_{e}$, by means of a quadrature, with the initial condition Eq. (71). In performing this quadrature we may use with advantage the fact that $T_{a} / T_{p}$ is large; $x_{p}$ or $T_{p}$ may then be taken as constant in Eq. (70) except in the exponential, which may be 
linearized. The resulting expression for $\tau$ as a function of $x_{p}$ is, for $x_{p}$ lower and not too close to $x_{i}$,

$$
\begin{aligned}
2(\sqrt{ } \tau & \left.-\sqrt{ } \tau_{i}\right)=\frac{2 T_{p}^{2} c_{p}}{T_{a} Q Y_{20}(1-\beta)} \\
& \times\left[2 y_{1 p}^{a} E_{p}^{-1}\left(\frac{c_{p} T_{p}}{Q Y_{20} T_{a}}\right)^{3}\left(\frac{T_{10}}{T_{p}}\right)^{a+3}\right. \\
& \left.\times \exp \left(\frac{T_{a}}{T_{10}}-\frac{T_{a}}{T_{p}}\right)\right]^{-1 / 2}
\end{aligned}
$$

The time required for the deflagration to move across the region " $x_{p}>x_{i}$ " is very short compared with time required for the motion of the flame across the region " $x_{p}<x_{i}$ ".

The oxidizer concentration ahead of the deflagration, $y_{1 p}$, goes to zero when the flame approaches the surface $x=x_{e}$, where the reactants are, before ignition, in stoichiomentric proportions. The reaction zone analysis, which led to Eq. (70), must then be substituted by an analysis in which both $y_{1}$ and $y_{2}$ are considered small, of order $T_{e} / T_{a}$, through the reaction zone. After crossing the surface $x=x_{\epsilon}$, the variations of $y_{2}$ across the reaction zone become small compared with its mean value; Eq. (70) may again be used to calculate the flame propagation velocity with the roles of $y_{1}$ and $y_{2}$ exchanged, and some minor additional modifications.

For this one-dimensional analysis to be directly applicable, the ignition source must also have a one-dimensional structure; however, the ideas used here could also be used to analyze the flame propagation process through a one-dimensional mixing layer, when the ignition source is point-like, as occurred in the experimental work of Liebman et al. (1970).

When ignition is associated not with an artificial source of heat but with chemical heat release, the ignition analysis of Section III, which resulted in thermal runaway at $\left(x_{I}, \tau_{I}\right)$, provides the initial condition for Eq. (70) if the difference between the initial temperatures of oxidizer and fuel is small $\left(\beta_{1} \sim 1\right)$. Equation (72) shows that the time required for the flame to move across the mixing layer is small compared with the ignition time, so that the factor $\tau$ appearing in the left hand side of Eq. (70) may be written as $\tau_{I}$ and consequently $2(\sqrt{ } \tau-$ $\left.\sqrt{ } \tau_{I}\right)$ may be replaced by $\left(\tau-\tau_{I}\right) / \sqrt{ } \tau_{I}$ in Eq. (72) for $x_{p}<x_{I}$.

The analysis of Section III provides the spontaneous ignition time $\tau_{I}$, for $1>\beta \sim 1$, as

$$
\tau_{I}=2 e^{-2}(2-\beta)^{-2}\left(1-T_{20} / T_{10}\right) \gamma_{11}^{2}\left(T_{a} / T_{10}\right)
$$

where

$$
\eta_{1}^{2}=\ln \left\{\left(T_{a} / T_{10}\right)\left(1-T_{20} / T_{10}\right)(1-\beta) / 4 \sqrt{ } \pi\right\}-\ln \eta_{1}
$$

but does not provide, unless $1-\beta \ll 1$, a similarly well defined $x_{I}$ to be used with $\tau_{I}$ as the initial condition for Eq. (70). This initial condition should be obtained from an analysis of a transition stage for $\tau$ close to $\tau_{I}$, in which the time derivative term left out of Eq. (38) should be retained. The time involved in this transition stage, and the time required for the flame to move across the mixing layer to the region where $T_{p}$ is again close to $T_{e}$, can be shown, by order of magnitude arguments, to be small, of order $1 / \eta_{1}{ }^{2}$ compared with the ignition time $\tau_{I}$.

When $1-\beta \ll 1$, Eq. (51) may be used to obtain the value of $x_{I}$ to use as the initial condition for Eq. (70), if the resulting value

$$
x_{I}=2(1-\beta)^{-1}\left(T_{10} / T_{a}\right)\left(1-T_{20} / T_{10}\right)^{-1}
$$

is small compared with one. Otherwise, we may use Eq. (61) for a more accurate determination of the ignition time $\tau_{I}$ and ignition spot $x_{I}$. Although the analysis leading to Eq. (61) was carried out under the assumption $\beta>1$, it is not difficult to show (by direct substitution, for example) that it can also be used to calculate the temperature distribution at times $\tau$ of order $(1-\beta)^{-2}$ prior to ignition, if $1-\beta \ll 1$. The lower branch of the $x_{p}(\tau)$ relation, resulting from Eq. (61), gives the position of the reaction zone up to times very close to the ignition time. The relation $x_{p}(\tau)$ given by Eq. (61) may become single-valued if $(1-\beta) T_{a} / T_{10}$ is not large enough; in this case Eq. (61) describes the continuous transition from frozen flow to equilibrium flow.

\section{$V$ THE DIFFUSION CONTROLLED STAGE}

When the premixed flame reaches the surface $x=x_{e}$, in the case $\beta>1$, or after crossing the surface $x=x_{e}$ in the case $\beta<1$, a thin reaction region is established around $x=x_{e}$, where the reactants burn after diffusing through the reaction products from both sides of the flame. This thin flame separates two regions which are under nearequilibrium conditions; one is without fuel and the other without oxidizer, in the first approximation. 
That is outside of the reaction zone, in first approximation,

$$
\begin{array}{lll}
y_{1}=0 & \text { for } & x>x_{e} \\
y_{2}=0 & \text { for } & x<x_{e}
\end{array}
$$

the concentration of the other reactant and the temperature are then obtained from Eqs. (15) and (16). Higher order approximations will introduce corrections of order $T_{e} / T_{a}$ to these values of $y_{1}$ and $y_{2}$, for appropriately large values of $\tau$.

For the analysis of the reaction zone following Liñan (1973), we introduce the variables $\Gamma$ and $Z$ defined by the relations,

$$
\begin{aligned}
& y_{1}=\left(T_{e} / T_{a}\right)(\Gamma-Z) / A \\
& y_{2}=\left(T_{e} / T_{a}\right)\left(Y_{10} / \nu Y_{20}\right)(\Gamma+Z) / A
\end{aligned}
$$

where the factor $T_{e} / T_{a}$ is included so as to make $\Gamma$ and $Z$ of order unity in the reaction zone. The parameter $\boldsymbol{A}$ will be chosen below, [Eq. (83)], so as to obtain a certain normalized equation and boundary conditions [Eqs. (84) and (87)] when calculating the first approximation for $\Gamma(z)$ for large values of $T_{a} / T_{e}$.

When analyzing the reaction zone we may, in first approximation, neglect the unsteady term in Eq. (18) and consider the factor $\exp \left(-2 \eta^{2}\right)$ as constant and equal to $\exp \left(-2 \eta_{e}{ }^{2}\right)$. This is equivalent to setting a balance between the chemical heat release and conduction normal to the reaction layer. In addition, the energy conservation equation may be further simplified, because the deviations, in the reaction zone of the temperature from the adiabatic flame value $T_{e}$ will be found to be small, of order $T_{e}^{2} / T_{a}$ at most. So that Eq. (18) reduces in the reaction layer in first approximation, to

$$
\begin{aligned}
\frac{\partial^{2} T}{\partial x^{2}}= & -\tau K_{e} T_{10}\left(T_{a} / T_{e}\right)^{a+b+1} \\
& \times y_{1}^{a} y_{2}^{b} \exp \frac{T_{a}\left(T-T_{e}\right)}{T_{e}^{2}}
\end{aligned}
$$

where

$$
\begin{aligned}
K_{e}= & 4 \pi \exp \left(2 \eta_{e}{ }^{2}\right)\left(\frac{T_{10}}{T_{a}}\right)^{a+b+1} \\
& \times\left(\frac{T_{e}}{T_{a}}\right) \exp \left(\frac{T_{a}}{T_{10}}-\frac{T_{a}}{T_{e}}\right)
\end{aligned}
$$

When Eq. (79) is written in terms of the variables $\Gamma$ and $Z$,

$$
\begin{aligned}
\Gamma=A( & \left.T_{a} / T_{e}\right)\left[\left(\nu c_{p} / Q Y_{10}\right)\left(T_{e}-T\right)\right. \\
& \left.-\left\{1+(2 \beta-1)\left(\nu Y_{20} / Y_{10}\right)\right\}\left(x-x_{e}\right) / 2\right]
\end{aligned}
$$

and

$$
Z=A\left(T_{a} / T_{e}\right)\left(1+\nu Y_{20} / Y_{10}\right)\left(x-x_{e}\right) / 2
$$

If we choose

$$
\begin{aligned}
A^{(1+a+b)}= & 4 \tau K_{e}\left(\frac{c_{p} T_{10}}{Q Y_{20}}\right)\left(\frac{\nu Y_{20}}{Y_{10}}\right)^{1-b} \\
& \times\left(1+\frac{\nu Y_{20}}{Y_{10}}\right)^{-2}
\end{aligned}
$$

we obtain the equation

$$
\Gamma_{Z Z}=(\Gamma-Z)^{a}(\Gamma+Z)^{b} \exp \{(\Gamma+g Z) / \Delta\},
$$

where

$$
g=\left\{1+(2 \beta-1) \nu Y_{20} / Y_{10}\right\}\left(1+\nu Y_{20} / Y_{10}\right)^{-1}
$$

and

$$
\Delta=A v c_{p} T_{e} / Q Y_{10}
$$

The matching conditions between the solution of Eq. (84) and the first approximation, Eqs. (75) and (76), for the solution in the outer regions, provide the boundary conditions for Eq. (84):

$$
\begin{array}{lll}
\Gamma_{Z}=-1 & \text { for } & Z \rightarrow-\infty, \\
\Gamma_{Z}=1 & \text { for } & Z \rightarrow \infty
\end{array}
$$

The function $\Gamma(Z, g, \Delta)$ was obtained by numerical integration of Eq. (84), for $a=b=1$, by L.iñán (1973). Only one solution exists for $g>1$ (or $\beta>1$ ) and all values of $\Delta$ : however, the resulting values of $\Gamma$ are large for $\Delta \ll 1$ and, therefore, the asymptotic analysis leading to Eq. (84) is then no longer valid. For $|g|<1$, there are two solutions for all values of $\Delta$ above a minimum value $\Delta_{m}(g)$ and no solutions for $\Delta<\Delta_{m}$. Figure 11 shows some typical results.

The asymptotic value of $(\Gamma-Z)_{\infty}$ is a measure of the deviation from equilibrium; it tends to zero for large values of $\Delta$ in one of the solutions, which is the only one with physical meaning. The fact that $(\Gamma-Z)_{\alpha}$ is different from zero indicates that there is leakage of oxidizer through the reaction zone. Figure (12) shows $(\Gamma-Z)_{\alpha}$ as a function of $\Delta$ for several values of $g$. 


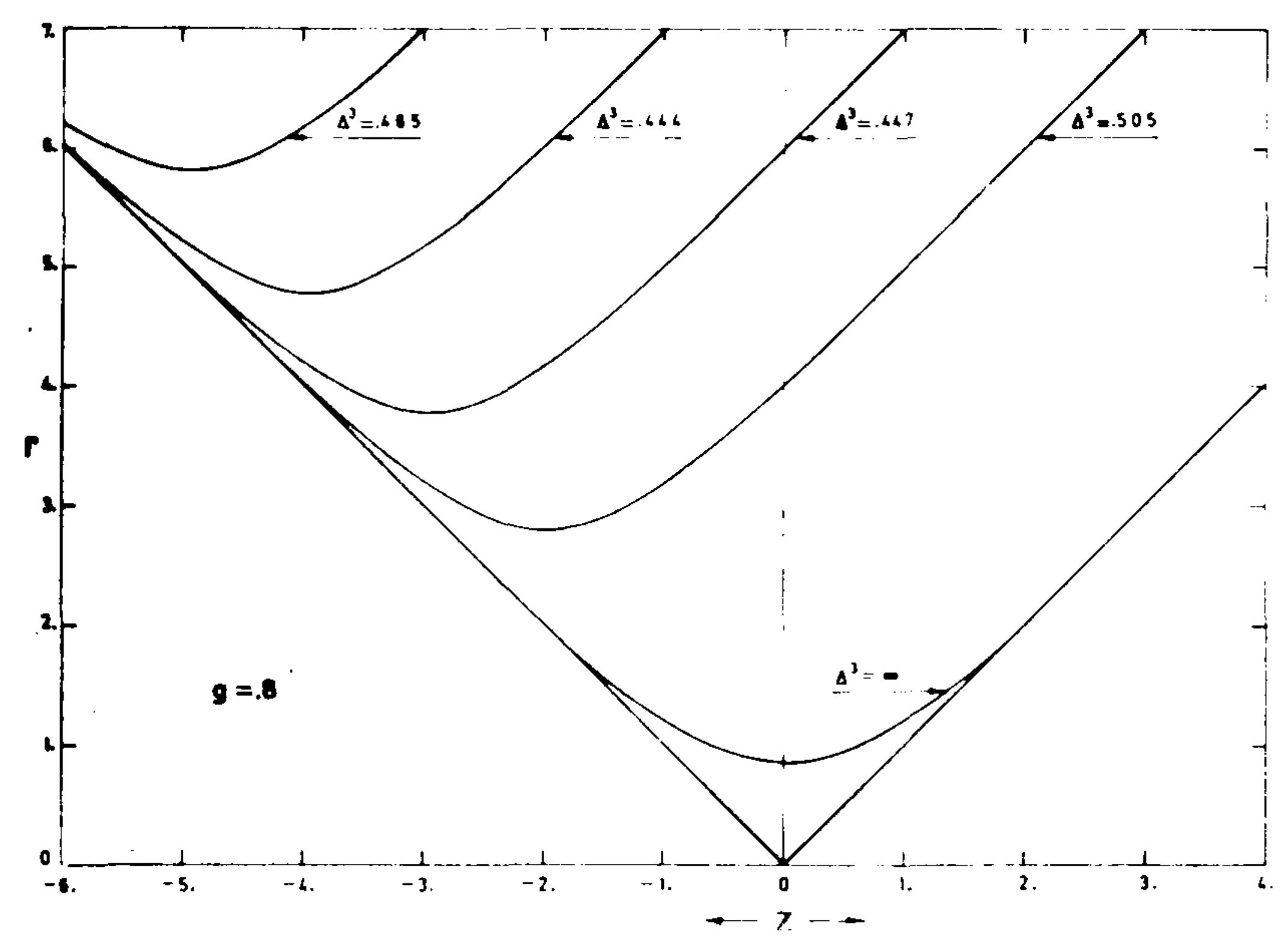

FIGURE 11 Internal structure of the reaction zone in the diffusion controlled stage, as resulting from the numerical solution of Eqs. (84) and (87) for $a=b=1$.

The numerical results for $\Delta_{m}(g)$ may be correlated, with errors of 1 percent, by the expression

$$
\begin{aligned}
\Delta_{m} 3 & =e(1-g) \\
& \times\left[1-(1-g)+0.26(1-g)^{2}+0.055(1-g)^{3}\right]
\end{aligned}
$$

which has the correct asymptotic form for smal ${ }^{1}$ values of $(1-g)$.

For large values of $\Delta, \Gamma(Z)$ is given by the solution of the equation

$$
\Gamma_{Z Z}=(\Gamma-Z)(\Gamma+Z)
$$

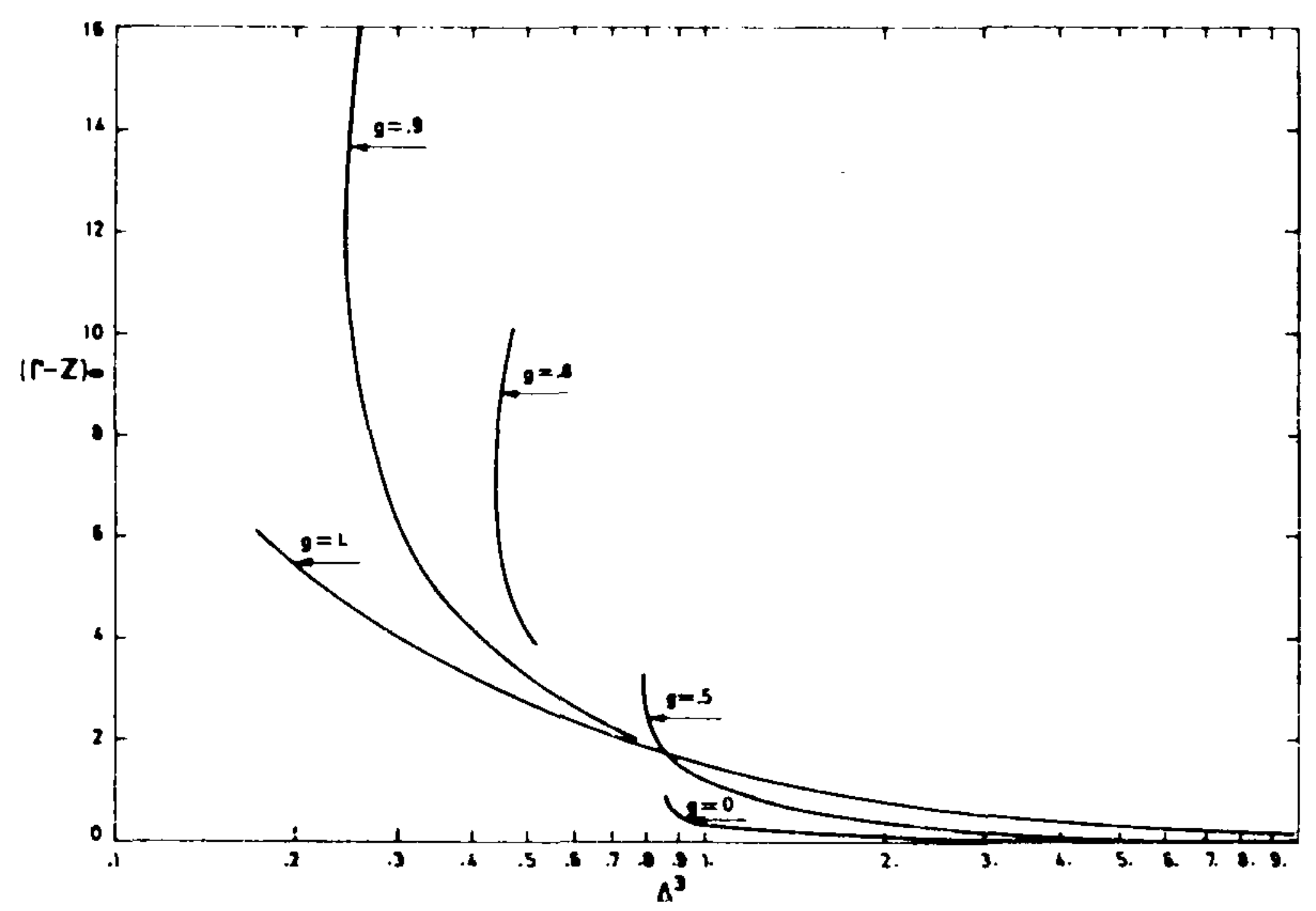

FIGURE 12 Leakage of oxidizer through the reaction zone as a function of the non-dimensional time $\Delta^{3}$, resulting from the quasi-steady thin diffusion flame analysis. which was obtained by Liñán (1961) when analyzing the diffusion flames structure for large reaction rates, with the boundary conditions Eq. (87).

A thin diffusion flame separating regions where the reactants do not coexist in first approximation, does not exist for $\beta<1$ if $\tau$ is lower than a minimum value, $\tau_{m}$, given by Eqs. (83), (86) and (88). However the resulting value of $\tau_{m}$ is very small compared with $\tau_{I}$ unless $1-\beta \ll 1$; the corresponding values of $\Delta$ are for $\tau>\tau_{i}$, very large compared with 1 , so that the reaction zone is extremely thin and its structure is described by Eq. (89) with the boundary condition (87).

For values of $\beta>1$ there is not an equivalent minimum value of $\Delta$ (or $\tau$ ), below which Eq. (84) would not have a solution with the boundary conditions, Eq. (87). However if $\Delta \ll 1$ the resulting values of $(\Gamma-Z)_{\infty}$ become so large compared with 1 , that this thin flame analysis is no longer valid, and it should be replaced by the flame propagation analysis of Section IV.

\section{LAMINAR MIXING AND CHEMICAL REACTION OF TWO PARALLEL STREAMS}

When studying the laminar mixing and simultaneous chemical reaction between two parallel streams of fuel and oxidizer, the boundary layer approximations may be used to simplify the problem, if the appropriate Peclet number, $V L / D$, based on the characteristic extent of the combustion zone in the streamwise direction is large. As a result of the neglect of upstream diffusion, under these approximations, the governing equations become parabolic, as occurs with the equations describing the evolution of unsteady mixing layers. See Williams (1965).

Marble and Adamson (1954) used the boundary layer approximations to analyze the process of ignition of a reactive stream by a hot parallel stream of its reaction products. It is clear that this problem, and the more general problem of chemical reaction between two parallel streams, admits an asymptotic treatment for large activation energies, following the ideas developed in the previous sections.

In particular, if both streams have equal initial velocities $V$, the equations describing their mixing and chemical reaction reduce exactly to the equations for the unsteady mixing layer, with $x_{1} / V$ 
replaced by $t$, if the boundary layer assumptions are applicable. The ignition delay length, $x_{1 I}$, may then be obtained from the ignition time, $t_{I}$, as $x_{1 I}=V t_{I}$; so that for the boundary layer approximations to be applicable, the Peclet number $V^{2} t_{I} / D$ must be large compared to 1 .

The flame propagation analysis for $\beta<1$, in Section IV, cannot be used to describe the deflagration waves originating in the ignition point unless the resulting flame propagation velocity is very small compared with $V$; in this case the flame is tilted downstream so much that only diffusion normal to the mixing layer is important. When the flame propagation velocity, corresponding to the local mixture, turns out to be comparable although smaller than the flow velocity, the boundary layer assumptions may still be used to calculate the ignition delay length. However to calculate the flame trajectory we can no longer use directly the analysis of Section IV because:

a) Diffusion normal to the flow direction should be replaced by diffusion normal to the flame in both the thin reaction layer and in its preceding thin transport layer.

b) The chemical heat release causes the density to decrease and, therefore, the mixture to expand if the mixing layer is unconfined; this expansion takes place. only crosswise to the flow direction if the boundary layer approximations are used. But, it is clear that the aerodynamic flow field induced by a premixed flame oblique to the flow direction includes large velocity perturbations both transverse and longitudinal to the original flow. A detailed analysis of this problem would, however, end up in showing that the streamwise extent of the region where the boundary layer approximations cannot be used is small, of the order of the thickness of the mixing layer at the ignition point, compared with the extent of the ignition region.

If, somewhere in the mixing region, the flame propagation velocity is larger than the flow velocity, the flame would move upstream after ignition, until it would sit at a distance, 1 , from the origin of the mixing layer such that $V 1 / D \sim 1$. The boundary layer assumptions cannot be used, in this case, to calculate the ignition length, or study the ignition region. However, at distances $x_{1}$ downstream such that $V x_{1} / D \ll 1$ the reaction becomes diffusion controlled, and the boundary layer assumptions together with the thin flame assumption can be used to describe this process.

\section{CONCLUSIONS AND GENERALIZATION}

We have found that several stages exist in the evolution of unsteady mixing layer between two gases undergoing an Arrhenius irreversible reaction with a large activation energy.

In a first "ignition" stage the chemical heat release is small so that the mixing appears to be frozen; however, it may cause the reaction rate to increase by a large factor during the last part of this stage, and thereby produce a thermal runaway, characteristic of ignition.

The character of the ignition stage, and the later evolution of the flame, depend on the value of the difference between the initial temperatures of the reactants, and on whether the final adiabatic flame temperature $T_{e}$ is larger or less than the maximum initial temperature, $T_{10}$.

A thermal runaway, characterizing an ignition time, does not exist if $T_{e}-T_{10}$ is sufficiently small or negative. In the other cases an ignition time may be defined, $t_{I}=t_{1} \tau_{I}$, where $t_{1}$ is given by Eq. (13), and $\tau_{I}$ is a function of

$$
\beta_{1}=\left(T_{10}-T_{20}\right)\left(T_{a} / T_{10}{ }^{2}\right)
$$

represented, for $a=b=1$, in Figure 7, for $\beta_{1}$ not too large. For $\beta_{1} \gg 1$, Eq. (73) is an approximate expression for the ignition time if $(1-\beta) T_{a} / T_{10}$ is large. $\dagger$

It should be noticed that the ignition time does not depend on the transport coefficients. It is of the order of the minimum ignition time that we would obtain in the mixing layer, when neglecting the effects of heat conduction and diffusion.

Local chemical equilibrium is established rapidly as a result of the thermal runaway. Figure 1 shows schematically the trajectories of the premixed flames occurring after spontaneous ignition. Figure 3 shows schematically the distributions of temperature in terms of the variable $x$ for different values of time corresponding to the various regimes. From these temperature profiles the reactant concentration profiles could be easily inferred, if Eqs. (15) and (16) are taken into account.

Figure 1a corresponds to the case $T_{10}-T_{20} \sim$ $T_{10}{ }^{2} / T_{a}$, when two classical deflagrations originate at the ignition point and move toward the edges of

\footnotetext{
$\dagger$ In this case the ignition time may also be inferred from expression (61) in the way described at the end of Section IV for $\beta$ close to one.
} 
the mixing layer where they slow down and thicken considerably. One of the flames leaves behind a thin diffusion flame when crossing the surface $x=x_{e}$, where the reactants were prior to ignition in stoichiometric proportions.

For large values of $T_{10}-T_{20}$ ignition occurs at the hotter edge of the mixing layer. If $T_{e}>T_{10}$, as represented in Figure $1 \mathrm{~b}$, a classical deflagration will then move rapidly across the mixing layer to burn completely the reactant locally in defect; again, after crossing the surface, $x=x_{e}$, a diffusion flame will be established there. The deflagration will slow and its preceding transport layer will thicken when the flame temperature decreases below the value $T_{10}$.

If $T_{e}<T_{10}$, as shown in Figure 1c, a thin flame originating at the hotter edge will move slowly across the mixing layer, with a transport layer ahead, covering the whole mixing layer, where the temperature and reactants have locally similar distributions. Upon reaching the surface $x=x_{e}$, the motion of the reaction layer ceases, and a thin diffusion flame is established, where, later, the reaction is diffusion controlled. The same type of result is obtained for small positive values of $T_{e}-T_{10}$ if the activation temperature is not too large. In these cases the transition from the frozen flow solution to the equilibrium solution is smooth, and is described by Eq. (61).

Because of the large number of non-dimensional parameters involved in this problem, we do not attempt in this paper a parametric analysis of the results.

The rapid transition from a nearly frozen form of mixing to a diffusion controlled mode of combustion, resulting from a local thermal runaway and a subsequent deflagration wave, will be found when analyzing a wide variety of problems of chemical reactions in unpremixed systems. It is clear that if an overall reaction rate is applicable and the activation energy is large, the methods and ideas developed here may be used to calculate the ignition time and the deflagration wave system which follows.

Many of these ideas may be used to cover more complex rate expressions. The extension to cases with more realistic transport coefficient is easy.

Finally these ideas can be used to simplify the analysis of turbulent mixing with chemical reactions in unpremixed systems. Mixing at the molecular scale must occur before combustion takes place in turbulent flow. Around each eddy of fuel a layer will exist, where mixing with the surrounding eddies of oxidizer takes place at the molecular scale. This mixing will be enhanced by the stretching of the mixing layer due to turbulence. However, it appears very likely that the ignition time for each of these layers will still be of the order of the one predicted here for the laminar case. Once ignition occurs, a deflagration wave will establish a diffusion flame surrounding each eddy of fuel; the subsequent turbulent combustion will be diffusion controlled, as in the analysis by O'Brien (1971) of turbulent mixing of two rapidly reacting species.

\section{ACKNOWLEDGEMENT}

The research reported here has been partially sponsored under ARO-INTA Consultant Services Subcontracts Nos. 71-14-TS/OMD and 72-13-TS/OMD. We thank Drs R. Rhodes and C. E. Peters of ARO, Inc., for the discussions with them on turbulent reacting flows, which provided the stimulus for carrying out this analysis of the transition from frozen to equilibrium flow. We wish to express also our appreciation for the many helpful discussions of this problem with Professor Forman Williams of La Jolla, and with $\mathrm{Dr}$ José L. Urrutia of INTA. We also thank Mr V. Torroglosa who carried out most of the numerical calculations.

\section{REFERENCES}

Brevig, O., and Shahrokhi, F. (1971). On the free turbulent mixing and combustion between coaxial hydrogen and air streams. 9th Aerospace Sciences Meeting, AIAA Paper 71-75.

Bush, W. B., and Fendell, F. E. (1970). Asymptotic analysis of laminar flame propagation for general Lewis numbers. Combustion Science and Technology, 1, 421.

Clarke, J. F. (1967a). The laminar diffusion flame in Oseen flow, Proc. of the Roy. Soc., Series A, 296, No. 1447, pp. 519-545.

Clarke, J. F. (1967b). The laminar diffusion flame behind a blunt body. A constant pressure Oseen-flow model. J. Inst. Maths. Applic., 3, 347.

Chung, P. M., Fendell, F. E., and Holt, J. F. (1966). Nonequilibrium anomalies in the development of diffusion flames. $A I A A J ., 4,1020$.

Fendell, F. E. (1965). Ignition and extinction of initially unmixed reactants. $J$. Fluid Mech., 21, 281.

Ferri, A. (1964). Review of problems in application of supersonic combustion. J. Roy. Astron. Soc., 64, No. 654 , pp. 575-597.

Friedlander, S. K., and Keller, K. H. (1963). The structure of the zone of diffusion controlled reaction. Chem. Eng. Sci., 18, 365.

Hermance, C. E., and Kumar, R. K. (1970). Gas phase ignition theory for homogeneous propellants under shock tube conditions. $A I A A J .8,1551$.

Jain, V. K., and Kumar, R. N. (1969). Theory of laminar flame propagation with non-normal diffusion. Combustion and Flame, 13, 285. 
Kashiwagi, T., and Summerfield, M. (1973). Ignition and flame spreading over a solid fuel. Non-similar theory for a hot oxidizing boundary layer. Fourteenth Symposuim (International) on Combustion, The Combustion Institute (Pittsburgh, Pa). 1235-1247.

Krishnamurty, L., and Williams, F. A. (1971). Kinetics and regression. Siam J. Appl. Math., 20, 590.

Libby, P. A., and Economos, E. (1963). A flame zone model for chemical reaction in a laminar boundary layer with application to the injection of hydrogen-oxygen mixtures. Int. Heat Mass Transfer, 6, 113.

Liebman, I., Corry, J., and Perlee, H. E. (1970). Flame propagation in layered methane-air systems. Combustion Science and Technology, 1, 257.

Lindberg, R. C., and Schmitz, R. A. (1969). On the multiplicity of steady states in boundary layer problems with surface reaction. Chem. Eng. Sci., 24, 1113.

Liñán, A. (1961). On the internal structure of laminar diffusion flames. OSR/EOAR TN 62-24, INTA, Madrid. Also ASTIA AD 273069.

Liñán, A. (1963). On the structure of laminar diffusion flames. Aero. Eng. Thesis, California Institute of Technology. Also, Tech. Rept. FM 63-2. INTA Madrid, Spain.

Liñán, A. (1970). Diffusion flames and supersonic combustion. Ann. Sci. Rept. No. 1 EOAR Cont. No. F6105269-0036. Madrid.

Liñan, A. (1973). The asymptotic structure of counter flow diffusion flames for large activation energies. Presented at the "Fourth International Colloquium on Gasdynamics of Explosions and Reactive Systems", La
Jolla, July 1973. Also (1974). Acta Astronautica, 1, 1007-1039.

Marble, F. E., and Adamson, T. C. Jr. (1954). Ignition and combustion in a laminar mixing region. Jet Propulsion, $24,85$.

O'Brien, E. E. (1971). Turbulent mixing of two rapidly reacting chemical species. Physics of Fluids, 14, 1326-31.

Pearson, J. R. A. (1963). Diffusion of one substance into a semi-infinite medium containing another with secondorder reaction. Appl. Sci. Res., A11, 321.

Strahle, W. C. (1969). A transient problem on the evaporation of a reactive fuel. Combustion Science and Technology, 1, 25.

Waldman, C. H., Cheng, S. I., Sirignano, W. A., and Summerfield, M. (1969). Theoretical studies of diffusion flame structures. AMS Report 860, Jan. 1969, Princeton Univ., Princeton, N.J. (Also C. H. Waldman, Ph.D. Thesis.)

Williams, F. A. (1965). Combustion Theory. Chapter 12 Addison-Wesley.

Wolanski, P., and Wójcicki, S. (1973). Investigation into the mechanism of the diffusion ignition of a combustible gas flowing into an oxidizing atmosphere. Fourteenth Symposium (International) on Combustion, The Combustion Institute (Pittsburgh, Pa.), 1217-1223.

Zeldovich, Y. B., and Frank-Kamenetsky, D. A., (1938). Theory of uniform flame propagation. Dokl. Akad. Nauk. SSSR, 19, 693.

Zeldovich, Y. B., and Frank-Kamenetsky, D. A. (1938). Theory of thermal flame propagation. J. Phys. Chem. Moscow, 12, 100. 